\title{
$M$ lump and interaction between $M$ lump and $N$ stripe for the third-order evolution equation arising in the shallow water
}

\author{
Onur Alp Ilhan', Jalil Manafian ${ }^{2 *}$ (D, As'ad Alizadeh ${ }^{3,4}$ and Sizar Abid Mohammed ${ }^{5}$
}

\section{${ }^{*}$ Correspondence:}

j_manafianheris@tabrizu.ac.ir

${ }^{2}$ Department of Applied

Mathematics, Faculty of

Mathematical Sciences, University

of Tabriz, Tabriz, Iran

Full list of author information is

available at the end of the article

\begin{abstract}
In this paper, we use the Hirota bilinear method for investigating the third-order evolution equation to determining the soliton-type solutions. The M lump solutions along with different types of graphs including contour, density, and three- and two-dimensional plots have been made. Moreover, the interaction between 1-lump and two stripe solutions and the interaction between 2-lump and one stripe solutions with finding more general rational exact soliton wave solutions of the third-order evaluation equation are obtained. We give the theorem along with the proof for the considered problem. The existence criteria of these solitons in the unidirectional propagation of long waves over shallow water are also demonstrated. Various arbitrary constants obtained in the solutions help us to discuss the graphical behavior of solutions and also grants flexibility in formulating solutions that can be linked with a large variety of physical phenomena. We further show that the assigned method is general, efficient, straightforward, and powerful and can be exerted to establish exact solutions of diverse kinds of fractional equations originated in mathematical physics and engineering. We have depicted the figures of the evaluated solutions to interpret the physical phenomena.
\end{abstract}

PACS Codes: 02.30.Jr; 05.45.Yv; 02.30.Ik

Keywords: Hirota bilinear method; Third-order evolution equation; M-lump solutions; Interaction; The unidirectional propagation; The existence criteria

\section{Introduction}

Some nonlinear waves in dynamical systems are of substantial importance and receive much attention, most particularly in the field of wave propagation in nonlinear systems [1]. They are expressed by nonlinear partial differential equations (NLPDEs) [2]. Application of nonlinear waves cuts across many fields, which include mixture of gas bubble in liquid [3], waves in elastic tubes [4], systems incorporating damping and dispersion [5], KP lump in ferrimagnets [6], chemical physics, and geochemistry [7]. However, the quest for exact explicit solutions of these equations remains a hot topic. Moreover, looking for localized solutions and, more specifically, solitary wave solutions, many methods of solving nonlinear wave developed by researchers over the years, including [8-16], lump-type

(c) The Author(s) 2020. This article is licensed under a Creative Commons Attribution 4.0 International License, which permits use sharing, adaptation, distribution and reproduction in any medium or format, as long as you give appropriate credit to the original author(s) and the source, provide a link to the Creative Commons licence, and indicate if changes were made. The images or other third party material in this article are included in the article's Creative Commons licence, unless indicated otherwise in a credit line to the material. If material is not included in the article's Creative Commons licence and your intended use is not permitted by statutory regulation or exceeds the permitted use, you will need to obtain permission directly from the copyright holder. To view a copy of this licence, visit http://creativecommons.org/licenses/by/4.0/. 
solutions [17-32], interaction soliton-soliton, soliton-kink, and kink-kink [33, 34], interactions between solitary wave solutions and lump solutions [35, 36], as well as periodic wave solutions [37-40] remain a very interesting subject for researchers. Several mathematical methods are used in the search for these solutions. For example, the exp-function method [41, 42], the homotopy perturbation technique [43], inverse scattering method [44], and so on.

The Hirota bilinear method for finding the lump soliton solutions and interaction of a lump solution with some of one-line, two-line, and three-line and even kink-breathersoliton solutions of evolution equations was initially introduced by Ma [45] by assuming the solution to be a series of functions including lump (combination of two positive functions as polynomial), lump-kink (combination of two positive functions as polynomial and exponential functions), called the interaction between a lump and one-line soliton, lump-soliton (combination of two positive functions as polynomial and hyperbolic cos functions), called the interaction between lump and two-line solitons, kinky breathersoliton (combination of two exponential functions and trigonometric cos function), and finally the stripe soliton function only with exponential solution function. The method received considerable attention and underwent through many improvements. It is important to note that the later improvements were given different names by different authors. For getting the lump solutions and their interactions, the authors have conjugated sufficient time to search the exact rational soliton solutions, for example, the KadomtsevPetviashvili (KP) equation [23], the B-Kadomtsev-Petviashvili equation [42], the reduced p-gKP and p-gbKP equations [25], the $(2+1)$-dimensional KdV equation [26], the $(2+1)$ dimensional generalized fifth-order KdV equation [33], the $(2+1)$-dimensional Burger equation [34], the nonlinear evolution equations [22], the generalized $(3+1)$-dimensional Shallow water-like equation [35], the $(2+1)$-dimensional Sawada-Kotera equation [30], and the $(2+1)$-dimensional bSK equation [31,32]. Various types of work for finding the periodic solitary wave solutions of the $(2+1)$-dimensional extended Jimbo-Miwa equations [37], interaction between lump and other kinds of solitary, periodic and kink solitons for the $(2+1)$-dimensional breaking soliton equation [27], lump and interaction between different types of those on the variable-coefficient Kadomtsev-Petviashvili equation [28], and periodic type and periodic cross-kink wave solutions [29] are achieved through the Hirota bilinear operator.

Nowadays NLPDEs have been created significant opportunity for the researchers to explain the tangible incidents. Therefore mathematicians and scientists work tirelessly to bring out different kinds of soliton solutions. As a result, in the past few years, several effective, rising, and realistic methods have been initiated and dilated to extract closed-form solutions to the NLPDEs, such as the generalized higher-order variable-coefficient Hirota equation [46], a higher-order nonlinear Schrödinger system [47], a (3 + 1)-dimensional Btype KP equation [48], the $(3+1)$-dimensional Zakharov-Kuznetsov-Burgers equation [49], the coherently coupled nonlinear Schrödinger equations [50], the coupled variablecoefficient fourth-order nonlinear Schrödinger equations [51], the $(2+1)$-dimensional Konopelchenko-Dubrovsky equations [52], a generalized KP equation [53], a (2+1)dimensional Davey-Stewartson system [54], and the $(2+1)$-dimensional generalized variable-coefficient KP-Burgers-type equation [55]. Various types of studies on solving NLPDEs were investigated by capable authors, for example, the space-time fractional nonlinear Schrödinger equation [56], the complex cubic-quintic Ginzburg-Landau equa- 
tion [57], symmetric nonlinear Schrödinger equations with the second- and fourth-order diffractions [58], the (2+1)-dimensional Korteweg-de Vries equation [59], and the $(1+1)$ dimensional coupled integrable dispersionless equations [60].

Take the third-order evolution equation of the form

$$
\mathbb{P}_{\mathrm{TOE}}(\Psi):=\Psi_{t}+\Psi_{x}+\frac{\alpha}{2}\left(3 \Psi \Psi_{x}+a \Psi_{y}\right)+\frac{\varepsilon}{6}(1-3 \tau) \Psi_{x x x}-\frac{\varepsilon}{4}(1+2 \tau) \Psi_{x y y}=0,
$$

where $\alpha, \varepsilon$ are small parameters, $\tau$ is the Bond number, and o $\Psi$ is the surface elevation in the $x$-direction, which is a model for the unidirectional propagation of long waves over shallow water, obtained via asymptotic expansion around simple wave motion of the Euler equations up to first-order in the small-wave amplitude [61, 62]. Assume the Hirota derivatives based on the functions $\rho(x)$ and $\varrho(x)$ given as

$$
\prod_{i=1}^{3} D_{\lambda_{i}}^{\pi_{i}} \rho . \varrho=\left.\prod_{i=1}^{3}\left(\frac{\partial}{\partial \lambda_{i}}-\frac{\partial}{\partial \mu_{i}}\right)^{o_{i}} \rho(\lambda) \varrho(\mu)\right|_{\mu=\lambda},
$$

where the vectors $\lambda=\left(\lambda_{1}, \lambda_{2}, \lambda_{3}\right), \mu=\left(\mu_{1}, \mu_{2}, \mu_{3}\right)$, and $o_{1}, o_{2}, o_{3}$ are arbitrary nonnegative integers. It is known that this third-order evolution equation possesses a Hirota bilinear form

$$
\begin{aligned}
\mathbb{B}_{\mathrm{TOE}}(\rho):= & \left(D_{x} D_{t}+D_{x}^{2}+\frac{a}{2} \alpha D_{y} D_{x}+\frac{\varepsilon}{6}(1-3 \tau) D_{x}^{4}-\frac{\varepsilon}{4}(1+2 \tau) D_{x}^{2} D_{y}^{2}\right) \rho . \rho \\
= & 2\left[\rho \rho_{x t}-\rho_{x} \rho_{t}+\rho \rho_{x x}-\rho_{x}^{2}+\frac{1}{2} a \alpha\left(\rho \rho_{x y}-\rho_{x} \rho_{y}\right)\right. \\
& +\frac{\varepsilon}{6}(1-3 \tau)\left(\rho \rho_{x x x x}-4 \rho_{x} \rho_{x x x}+3 \rho_{x x}^{2}\right) \\
& \left.-\frac{\varepsilon}{4}(1+2 \tau)\left(\rho \rho_{x x y y}-2 \rho_{x} \rho_{x y y}+\rho_{x x} \rho_{y y}-2 \rho_{y} \rho_{x x y}+2 \rho_{x y}^{2}\right)\right] .
\end{aligned}
$$

We utilize the following relation between the functions $\phi(x, y, t)$ and $\Psi(x, y, t)$ :

$$
\Psi(x, y, t)=R(\ln \rho(x, y, t))_{x}
$$

where $R=\frac{1}{18} \frac{\varepsilon\left(6 \tau\left(k_{1}^{3}+k_{1} k_{2}^{2}\right)+3 k_{1} k_{2}^{2}-2 k_{1}^{3}-6 a \alpha k_{2}-\left(k_{3}-k_{1}\right)\right)}{\alpha k_{1}^{2}}$. Based on the Bell polynomial theories of soliton equations, we get to the relation

$$
\mathbb{P}_{\mathrm{TOE}}(\Psi)=\left[\frac{\mathbb{B}_{\mathrm{TOE}}(\rho)}{\rho}\right]_{x} .
$$

Theorem 1.1 $\Psi=R(\ln \rho)_{x}$ is a solution to Eq. (1.1) if and only if $\rho$ satisfies the equation

$$
\begin{aligned}
\left(D_{x} D_{t}+D_{x}^{2}+\frac{a}{2} \alpha D_{y} D_{x}+\frac{\varepsilon}{6}(1-3 \tau) D_{x}^{4}-\frac{\varepsilon}{4}(1+2 \tau) D_{x}^{2} D_{y}^{2}\right) \rho . \rho \\
=2\left[\rho \rho_{x t}-\rho_{x} \rho_{t}+\rho \rho_{x x}-\rho_{x}^{2}+\frac{1}{2} a \alpha\left(\rho \rho_{x y}-\rho_{x} \rho_{y}\right)\right. \\
\quad+\frac{\varepsilon}{6}(1-3 \tau)\left(\rho \rho_{x x x x}-4 \rho_{x} \rho_{x x x}+3 \rho_{x x}^{2}\right) \\
\left.\quad-\frac{\varepsilon}{4}(1+2 \tau)\left(\rho \rho_{x x y y}-2 \rho_{x} \rho_{x y y}+\rho_{x x} \rho_{y y}-2 \rho_{y} \rho_{x x y}+2 \rho_{x y}^{2}\right)\right] .
\end{aligned}
$$


Proof Denoting $\Gamma=\partial_{x}(\ln \rho)$, from expression (1.4) we get

$$
\Psi=R \Gamma \quad \Longleftrightarrow \quad \rho=\exp \left(\frac{1}{R} \int \Psi d x\right)
$$

Then, by considering $\rho=\exp \left(\partial_{x}^{-1} \Gamma\right)>0$, the derivatives $\rho_{x}, \rho_{y}, \rho_{t}, \rho_{x x}, \rho_{y y}, \rho_{x y}, \rho_{x t}, \rho_{x x y}$, $\rho_{x y y}, \rho_{x x x}, \rho_{x x y y}$, and $\rho_{x x x x}$ can be written as

$$
\begin{aligned}
\rho_{x}= & \Gamma \exp \left(\partial_{x}^{-1} \Gamma\right), \\
\rho_{y}= & \partial_{x}^{-1} \Gamma_{y} \exp \left(\partial_{x}^{-1} \Gamma\right), \\
\rho_{t}= & \partial_{x}^{-1} \Gamma_{t} \exp \left(\partial_{x}^{-1} \Gamma\right), \\
\rho_{x x}= & \left(\Gamma^{2}+\Gamma_{x}\right) \exp \left(\partial_{x}^{-1} \Gamma\right), \\
\rho_{y y}= & \left(\left(\partial_{x}^{-1} \Gamma_{y}\right)^{2}+\partial_{x}^{-1} \Gamma_{y y}\right) \exp \left(\partial_{x}^{-1} \Gamma\right), \\
\rho_{x y}= & \left(\Gamma \partial_{x}^{-1} \Gamma_{y}+\theta_{y}\right) \exp \left(\partial_{x}^{-1} \Gamma\right), \\
\rho_{x t}= & \left(\Gamma \partial_{x}^{-1} \Gamma_{t}+\Gamma_{t}\right) \exp \left(\partial_{x}^{-1} \Gamma\right), \\
\rho_{x x x}= & \left(\Gamma^{3}+3 \Gamma \Gamma_{x}+\Gamma_{x x}\right) \exp \left(\partial_{x}^{-1} \Gamma\right), \\
\rho_{x x y}= & {\left[\left(\Gamma^{2}+\Gamma_{x}\right) \partial_{x}^{-1} \Gamma_{y}+2 \Gamma \Gamma_{y}+\Gamma_{x y}\right] \exp \left(\partial_{x}^{-1} \Gamma\right), } \\
\rho_{x y y}= & {\left[\Gamma\left(\partial_{x}^{-1} \Gamma_{y}\right)^{2}+\Gamma \partial_{x}^{-1} \Gamma_{y y}+2 \Gamma_{y} \partial_{x}^{-1} \Gamma_{y}+\Gamma_{y y}\right] \exp \left(\partial_{x}^{-1} \Gamma\right), } \\
\rho_{x x x x}= & {\left[\Gamma^{4}+6 \Gamma^{2} \Gamma_{x}+4 \Gamma \Gamma_{x x}+3\left(\Gamma_{x}\right)^{2}+\Gamma_{x x x}\right] \exp \left(\partial_{x}^{-1} \Gamma\right), } \\
\rho_{x x y y}= & {\left[\left(\Gamma^{2}+\Gamma_{x}\right)\left(\left(\partial_{x}^{-1} \Gamma_{y}\right)^{2}+\partial_{x}^{-1} \Gamma_{y y}\right)+4 \Gamma \Gamma_{y} \partial_{x}^{-1} \Gamma_{y}\right.} \\
& \left.+2 \Gamma_{x y} \partial_{x}^{-1} \Gamma_{y}+2 \Gamma \Gamma_{y y}+2\left(\Gamma_{y}\right)^{2}+\Gamma_{x y y}\right] \exp \left(\partial_{x}^{-1} \Gamma\right) .
\end{aligned}
$$

Plugging (1.8)-(1.19) into (1.3) yields the bilinear form of Eq. (1.3):

$$
\begin{aligned}
2\left[\rho \rho_{x t}-\rho_{x} \rho_{t}+\rho \rho_{x x}-\rho_{x}^{2}+\frac{1}{2} a \alpha\left(\rho \rho_{x y}-\rho_{x} \rho_{y}\right)+\frac{\varepsilon}{6}(1-3 \tau)\left(\rho \rho_{x x x x}-4 \rho_{x} \rho_{x x x}+3 \rho_{x x}^{2}\right)\right. \\
\left.\quad-\frac{\varepsilon}{4}(1+2 \tau)\left(\rho \rho_{x x y y}-2 \rho_{x} \rho_{x y y}+\rho_{x x} \rho_{y y}-2 \rho_{y} \rho_{x x y}+2 \rho_{x y}^{2}\right)\right] \\
=-\frac{1}{6} \exp \left(2 \partial_{x}^{-1} \Gamma\right)\left[12 \int \frac{\mathrm{d}^{2}}{\mathrm{~d} y^{2}} \Gamma \mathrm{d} x\left(\frac{\mathrm{d}}{\mathrm{d} x} \Gamma\right) \epsilon \tau+24\left(\frac{\mathrm{d}}{\mathrm{d} y} \Gamma\right)^{2} \epsilon \tau\right. \\
\quad+36\left(\frac{\mathrm{d}}{\mathrm{d} x} \Gamma\right)^{2} \epsilon \tau+6\left(\frac{\mathrm{d}^{3}}{\mathrm{~d} x^{3}} \Gamma\right) \epsilon \tau+6 \int \frac{\mathrm{d}^{2}}{\mathrm{~d} y^{2}} \Gamma \mathrm{d} x\left(\frac{\mathrm{d}}{\mathrm{d} x} \Gamma\right) \epsilon \\
\quad+6\left(\frac{\mathrm{d}^{3}}{\mathrm{~d} x \mathrm{~d} y^{2}} \Gamma\right) \epsilon \tau+12\left(\frac{\mathrm{d}}{\mathrm{d} y} \Gamma\right)^{2} \epsilon-6 a \alpha \frac{\mathrm{d}}{\mathrm{d} y} \Gamma-12\left(\frac{\mathrm{d}}{\mathrm{d} x} \theta\right)^{2} \epsilon \\
\left.\quad-2\left(\frac{\mathrm{d}^{3}}{\mathrm{~d} x^{3}} \Gamma\right) \epsilon+3\left(\frac{\mathrm{d}^{3}}{\mathrm{~d} x \mathrm{~d} y^{2}} \Gamma\right) \epsilon-12 \frac{\mathrm{d}}{\mathrm{d} t} \Gamma-12 \frac{\mathrm{d}}{\mathrm{d} x} \Gamma\right] \\
=-\frac{1}{6} \phi^{2}\left[12 \epsilon \tau \Gamma_{x} \partial_{x}^{-1} \Gamma_{y y}+24 \epsilon \tau\left(\Gamma_{y}\right)^{2}+36 \epsilon \tau\left(\Gamma_{x}\right)^{2}+6 \epsilon \tau \Gamma_{x x x}+6 \epsilon \Gamma_{x} \partial_{x}^{-1} \Gamma_{y y}\right. \\
\quad+6 \epsilon \tau \Gamma_{x y y}+12 \epsilon\left(\Gamma_{y}\right)^{2}-6 a \alpha \Gamma_{y}-12 \epsilon\left(\Gamma_{x}\right)^{2}-2 \epsilon \Gamma_{x x x} \\
\left.+3 \epsilon \Gamma_{x y y}-12 \Gamma_{t}-12 \Gamma_{x}\right],
\end{aligned}
$$


which can be rewritten as

$$
\begin{gathered}
\frac{1}{\phi^{2}}\left[\rho \rho_{x t}-\rho_{x} \rho_{t}+\rho \rho_{x x}-\rho_{x}^{2}+\frac{1}{2} a \alpha\left(\rho \rho_{x y}-\rho_{x} \rho_{y}\right)+\frac{\varepsilon}{6}(1-3 \tau)\left(\rho \rho_{x x x x}-4 \rho_{x} \rho_{x x x}+3 \rho_{x x}^{2}\right)\right. \\
\left.-\frac{\varepsilon}{4}(1+2 \tau)\left(\rho \rho_{x x y y}-2 \rho_{x} \rho_{x y y}+\rho_{x x} \rho_{y y}-2 \rho_{y} \rho_{x x y}+2 \rho_{x y}^{2}\right)\right] \\
=\frac{\left(D_{x} D_{t}+D_{x}^{2}+\frac{a}{2} \alpha D_{y} D_{x}+\frac{\varepsilon}{6}(1-3 \tau) D_{x}^{4}-\frac{\varepsilon}{4}(1+2 \tau) D_{x}^{2} D_{y}^{2}\right) \rho . \rho}{2 \rho^{2}}
\end{gathered}
$$

where $\Gamma=\frac{1}{R} \Psi=\partial_{x}(\ln \rho)$ and $\partial_{x}^{-1}(\cdot)=\int(\cdot) d x$. Therefore Eq. (1.21) is the third-order evolution-type equation, and the theorem has been proved.

We clearly confirm that other published papers do not cover ours, and made work is really new. Here our purpose is discovering the exact solutions of the third-order evaluation equation under consideration by the Hirota bilinear method for gaining the $M$ lump, the interaction between 1-lump and two-stripe solutions, and the interaction between 2lump and one-stripe solutions, which arise in more classes. We give a discussion about the third-order evaluation equation and the Hirota bilinear method. We also offer graphical illustrations of some solutions of the considered model along with the obtained solutions. After that, we deal with the probe of solutions and finish by conclusion.

\section{New M-lump solutions of the third-order evolution equation}

According to analysis in [39], based on the Hirota operator, the solution of nonlinear differential equation (1.3) can be written as

$$
\rho=\rho_{N}=\sum_{\sigma=0,1} \exp \left(\sum_{i<j}^{N} \sigma_{i} \sigma_{j} F_{i j}+\sum_{i=1}^{N} \sigma_{i} \eta_{i}\right),
$$

where

$$
\begin{aligned}
& \eta_{i}=k_{i}\left(x+p_{i} y-\left[1+\frac{1}{2} a \alpha p_{i}-\frac{1}{4} \epsilon k_{i}^{2} p_{i}^{2}(2 \tau+1)-\frac{1}{6} \epsilon k_{i}^{2}(3 \tau-1)\right] t\right)+\eta_{i}^{(0)}, \\
& \exp F_{i j}=\frac{\left(k_{i}-k_{j}\right)\left(2\left(k_{i}-k_{j}\right)\left(2 \tau p_{i} p_{j}+3 \epsilon \tau+p_{i} p_{j}-\epsilon\right)+\left(k_{i} p_{i}{ }^{2}-k_{j} p_{j}^{2}\right)(2 \tau+1)\right)}{\left(k_{i}+k_{j}\right)\left(2\left(k_{i}+k_{j}\right)\left(2 \tau p_{i} p_{j}+3 \epsilon \tau+p_{i} p_{j}-\epsilon\right)+\left(k_{i} p_{i}{ }^{2}+k_{j} p_{j}{ }^{2}\right)(2 \tau+1)\right)} .
\end{aligned}
$$

The notation $\sigma=0,1$ shows summation over all conceivable compositions of $\sigma_{1}=0,1, \sigma_{2}=$ $0,1, \ldots, \sigma_{N}=0,1$; the summation $\sum_{i<j}^{N}$ is over all conceivable compositions of $N$ values of $i<j$. For example, the first three expressions of (2.1) are as follows:

$$
\begin{aligned}
& \rho_{1}=1+e^{\eta_{1}}, \\
& \rho_{2}=1+e^{\eta_{1}}+e^{\eta_{2}}+a_{12} e^{\eta_{1}+\eta_{2}}, \\
& \rho_{3}=1+e^{\eta_{1}}+e^{\eta_{2}}+e^{\eta_{3}}+a_{12} e^{\eta_{1}+\eta_{2}}+a_{13} e^{\eta_{1}+\eta_{3}}+a_{23} e^{\eta_{2}+\eta_{3}}+a_{12} a_{13} a_{23} e^{\eta_{1}+\eta_{2}+\eta_{3}},
\end{aligned}
$$


where $a_{i j}=\exp \left(F_{i j}\right), i<j$. To find $M$ lump solutions of Eq. (1.1), by catching $\exp \left(\eta_{i}^{(0)}\right)=-1$ in (2.2), $\rho_{N}$ will be as follows:

$$
\rho_{N}=\sum_{\sigma=0,1}\left(\prod_{i=1}^{N}(-1)^{\sigma_{i}} \exp \left(\sigma_{i} \zeta_{i}\right) \prod_{i<j}^{N} \exp \left(\sigma_{i} \sigma_{j} F_{i j}\right)\right)
$$

where

$$
\zeta_{i}=k_{i}\left(x+p_{i} y-\left[1+\frac{1}{2} a \alpha p_{i}-\frac{1}{4} \epsilon k_{i}^{2} p_{i}^{2}(2 \tau+1)-\frac{1}{6} \epsilon k_{i}^{2}(3 \tau-1)\right] t\right) .
$$

Taking the limit as $k_{i} \rightarrow 0$ with all the $k_{i}$ of the same asymptotic order, we get:

$$
\rho_{N}=\sum_{\sigma=0,1}\left(\prod_{i=1}^{N}(-1)^{\sigma_{i}}\left(1+\sigma_{i} k_{i} \xi_{i}\right) \prod_{i<j}^{N}\left(1+\sigma_{i} \sigma_{j} k_{i} k_{j} G_{i j}\right)\right)+O\left(k^{N+1}\right) .
$$

In (2.8), we can see that $\rho_{N}$ is factorized by $\prod_{i=1}^{N} k_{i}$. By transformation $\Psi=R(\ln \rho)_{x}$ we get a rational solution of Eq. (1.5). The reduced $\phi_{N}$ is

$$
\begin{aligned}
\rho_{N}= & \prod_{i=1}^{N} \xi_{i}+\frac{1}{2} \sum_{i, j}^{N} G_{i j} \prod_{l \neq i, j}^{N} \xi_{l}+\frac{1}{2 ! 2^{2}} \sum_{i, j, s, r}^{N} G_{i j} G_{s r} \prod_{l \neq i, j, s, r}^{N} \xi_{l}+\cdots \\
& +\frac{1}{M ! 2^{M}} \sum_{i, j, \ldots, m, n}^{N} G_{i j} G_{k l} \cdots G_{m n} \prod_{p \neq i, j, k, l, \ldots, m, n}^{N} \xi_{p},
\end{aligned}
$$

where

$$
\xi_{i}=x+p_{i} y-\left(1+\frac{1}{2} a \alpha p_{i}\right) t
$$

Analytical behavior of the solution function $\rho_{1}$ as a 1-soliton is presented with $p_{1}=a+i b$ in Fig. 1. From (2.9) we usually get a singular solution. As regards, if we take $p_{M+i}=p_{i}^{*}(i=$ $1,2, \ldots, M)$ for $N=2 M$, then we obtain a class of nonsingular rational solutions called $M$ lump solutions, which were established in [17]. Continuing, we bring 1-lump and multiplelump solutions of Eq. (1.1) in the following subsections.
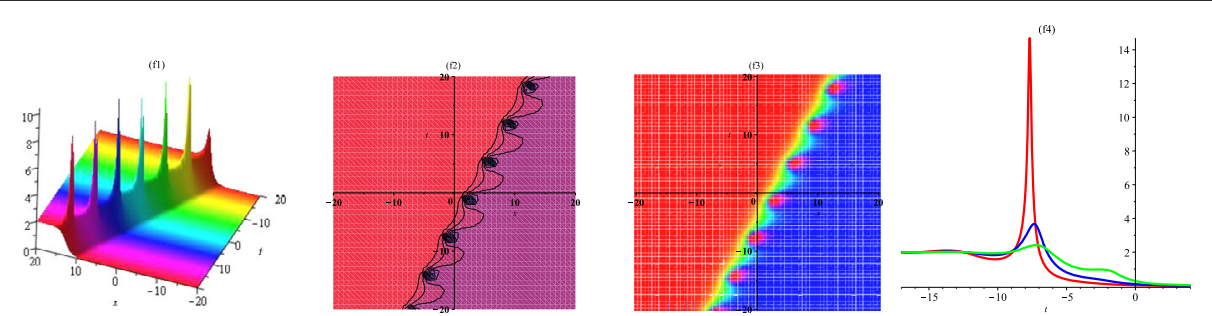

Figure 1 Diagram of 1-waves (2.4) using values $a=1.5, b=1, k_{1}=1, \alpha=0.2, \epsilon=0.3, \tau=2, \eta_{0}=0, R=2$, $y=-2$. (f1) 3D plot, (f2) contour plot, (f3) density plot, and (f4) $2 \mathrm{D}$ plot with (red $x=-1$, blue $x=0$, and green $x=1$ ) 


\subsection{1-lump solutions for Equation (1.1)}

By considering $\exp \left(\eta_{i}^{(0)}\right)=-1, i=1,2$, 1-lump solutions of equation (1.1) for discovering 2 -soliton solutions can be found in the form

$$
\rho_{2}=1-e^{\zeta_{1}}-e^{\zeta_{2}}+e^{\zeta_{1}+\zeta_{2}+F_{12}}
$$

where

$$
\begin{aligned}
\zeta_{i} & =k_{i}\left(x+p_{i} y-\left[1+\frac{1}{2} a \alpha p_{i}-\frac{1}{4} \epsilon k_{i}^{2} p_{i}^{2}(2 \tau+1)-\frac{1}{6} \epsilon k_{i}^{2}(3 \tau-1)\right] t\right), \quad i=1,2, \\
\exp & F_{12} \\
& =\frac{\left(k_{1}-k_{2}\right)\left(2\left(k_{1}-k_{2}\right)\left(2 \tau p_{1} p_{2}+3 \epsilon \tau+p_{1} p_{2}-\epsilon\right)+\left(k_{1} p_{1}^{2}-k_{2} p_{2}^{2}\right)(2 \tau+1)\right)}{\left(k_{1}+k_{2}\right)\left(2\left(k_{1}+k_{2}\right)\left(2 \tau p_{1} p_{2}+3 \epsilon \tau+p_{1} p_{2}-\epsilon\right)+\left(k_{1} p_{1}^{2}+k_{2} p_{2}^{2}\right)(2 \tau+1)\right)} \\
& =1-\frac{2 k_{1} k_{2}\left(\left(p_{1}^{2}+4 p_{1} p_{2}+p_{2}^{2}\right)(2 \tau+1)+4(3 \tau-1) \epsilon\right)}{\left(k_{1}+k_{2}\right)\left(\left(k_{1} p_{1}^{2}+2 k_{1} p_{1} p_{2}+2 k_{2} p_{1} p_{2}+k_{2} p_{2}^{2}\right)(2 \tau+1)+2 \epsilon\left(k_{1}+k_{2}\right)(3 \tau-1)\right)}
\end{aligned}
$$

and

$$
\begin{aligned}
\rho_{2}= & 1-e^{\zeta_{1}}-e^{\zeta_{2}}+e^{\zeta_{1}+\zeta_{2}} \\
& -\frac{2 k_{1} k_{2}\left(\left(p_{1}^{2}+4 p_{1} p_{2}+p_{2}^{2}\right)(2 \tau+1)+4(3 \tau-1) \epsilon\right) e^{\zeta_{1}+\zeta_{2}}}{\left(k_{1}+k_{2}\right)\left(\left(k_{1} p_{1}^{2}+2 k_{1} p_{1} p_{2}+2 k_{2} p_{1} p_{2}+k_{2} p_{2}^{2}\right)(2 \tau+1)+2 \epsilon\left(k_{1}+k_{2}\right)(3 \tau-1)\right)} .
\end{aligned}
$$

By considering the "long wave" limit as $k_{i} \rightarrow 0$ for $i=1,2$ with $\frac{k_{1}}{k_{2}}=2$, we conclude

$$
\rho_{2}=1-e^{\zeta_{1}}-e^{\zeta_{2}}+\left(\frac{1}{3} \frac{\left(2 p_{1}^{2}+2 p_{1} p_{2}-p_{2}^{2}\right)(2 \tau+1)+2(3 \tau-1) \epsilon}{\left(2 p_{1}^{2}+6 p_{1} p_{2}+p_{2}^{2}\right)(2 \tau+1)+6(3 \tau-1) \epsilon}\right) e^{\zeta_{1}+\zeta_{2}}
$$

where

$$
\zeta_{j}=x+p_{j} y-\left(1+\frac{1}{2} a \alpha p_{j}\right) t, \quad j=1,2
$$

Putting $p_{2}=p_{1}^{*}=a-i b$ into (2.13) and (2.14), we attain a nonsingular solution

$$
\begin{aligned}
\rho_{2}= & 1-e^{\zeta_{1}}-e^{\zeta_{2}} \\
& +\left(\frac{1}{3} \frac{\left(6 i a b+3 a^{2}+b^{2}\right)(2 \tau+1)+2(3 \tau-1) \epsilon}{\left(2 i a b+9 a^{2}+3 b^{2}\right)(2 \tau+1)+6(3 \tau-1) \epsilon}\right) e^{\zeta_{1}+\zeta_{2}}, \quad i=\sqrt{-1},
\end{aligned}
$$

where

$$
\begin{aligned}
& \zeta_{1}=x+(a+i b) y-\left(1+\frac{1}{2} a \alpha(a+i b)\right) t \\
& \zeta_{2}=x+(a-i b) y-\left(1+\frac{1}{2} a \alpha(a-i b)\right) t .
\end{aligned}
$$


Plugging (2.17) and (2.18) into $\Psi=R\left(\ln \rho_{2}\right)_{x}$ and putting $p_{1}=a+b i$, we obtain

$$
\begin{aligned}
\Psi= & -2 \Gamma_{1} R \\
& \times \frac{\left(\left(6 i a b+3 a^{2}+b^{2}\right)(2 \tau+1)+2 \epsilon \Gamma_{1}(3 \tau-1)\right) \Gamma_{1}-3\left(\left(2 i a b+9 a^{2}+3 b^{2}\right)(2 \tau+1)+6(3 \tau-1) \epsilon\right) \Gamma_{5}}{\Gamma_{6}-\left(\left(6 i a b+3 a^{2}+b^{2}\right)(2 \tau+1)+2(3 \tau-1) \epsilon\right) \Gamma_{1}^{2}+3\left(\left(2 i a b+9 a^{2}+3 b^{2}\right)(2 \tau+1)+6(3 \tau-1) \epsilon\right)\left(\Gamma_{3}+\Gamma_{4}\right)},
\end{aligned}
$$

$$
\begin{aligned}
& \Gamma_{1}=\mathrm{e}^{x+a y-t-\frac{1}{2} t a^{2} \alpha}, \quad \Gamma_{2}=\mathrm{e}^{x+a y-t-\frac{1}{2} t a^{2} \alpha}, \\
& \Gamma_{3}=\mathrm{e}^{x+i b y+a y-t-\frac{1}{2} i t a \alpha b-\frac{1}{2} t a^{2} \alpha}, \quad \Gamma_{4}=\mathrm{e}^{x-i b y+a y-t+\frac{1}{2} i t a \alpha b-\frac{1}{2} t a^{2} \alpha}, \\
& \Gamma_{5}=\cos \left(\frac{1}{2} b(t a \alpha-2 y)\right), \quad \Gamma_{6}=-3\left(2 i a b+9 a^{2}+3 b^{2}\right)(2 \tau+1)-18(3 \tau-1) \epsilon .
\end{aligned}
$$

We can see that solution (2.17) decays as $|x| \rightarrow \infty$ with amplitude $2 R$. In Figs. 1-2, solution (2.17) is plotted for an appropriate choice of diverse values and $\rho$ at space $x=-1,0,1$. From (2.17) we see that $\rho_{2}$ is a positive quadratic function compatible with the findings in [19]. By selecting suitable values of the parameters $p_{1}=a+i b$ and $p_{2}=a-i b$ the analytical treatment of 1-lump solution is presented in Fig. 2, including the 3D plot, density plot, and 2D plot when three spaces arise at spaces $x=-1, x=0$, and $x=1$. Also, choosing suitable values of the parameters $p_{1}=a+i b, p_{2}=c+i d$, the graphic representation of 1lump solution is presented in Fig. 3, including the 3D plot, density plot, and 2D plot when three spaces arise at spaces $x=-1, x=0$, and $x=1$. Likewise, selecting suitable values of the parameters $p_{1}=a+i b$ and $p_{2}=2$, the analytical treatment of 1-lump solution is presented in Fig. 4, including the 3D plot, density plot, and 2D plot when three spaces arise at spaces $x=-1, x=0$, and $x=1$. Finally, selecting suitable values of the parameters $p_{1}=3$ and $p_{2}=2$, the graphic representation of 1-lump solution is presented in Fig. 5,
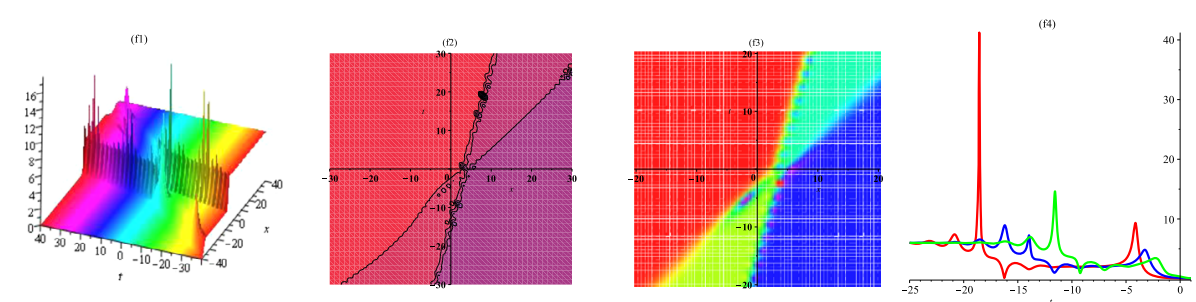

Figure 2 Diagram of 1-lump (2.17) by taking $a=1.5, b=1, k_{1}=1, k_{2}=2, \alpha=1, \epsilon=-1, \tau=2, \eta_{10}=\eta_{20}=0$, $R=2, y=-2$ at space (red $x=-1$, blue $x=0$, and green $x=1$ )
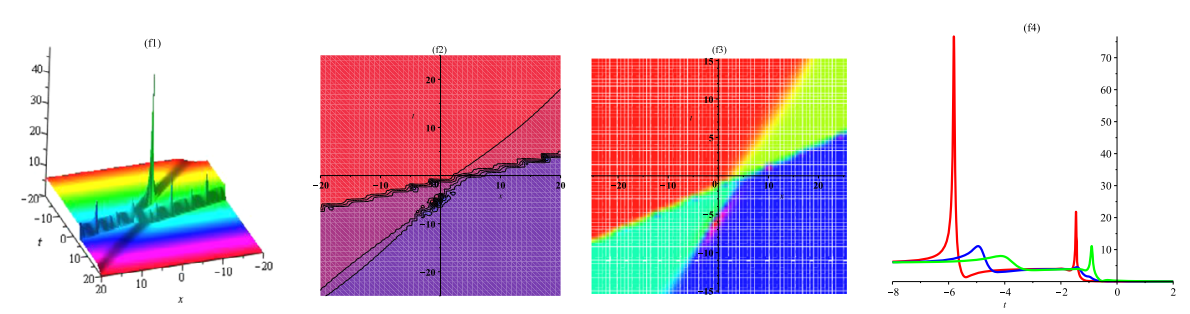

Figure 3 Diagram of 1-lump (2.17) by taking $a=1.5, b=1, c=2, d=3, k_{1}=1, k_{2}=2, \alpha=1, \epsilon=-1, \tau=2$, $\eta_{10}=\eta_{20}=0, R=2, y=-2$ at space $($ red $x=-1$, blue $x=0$, and green $x=1)$ 

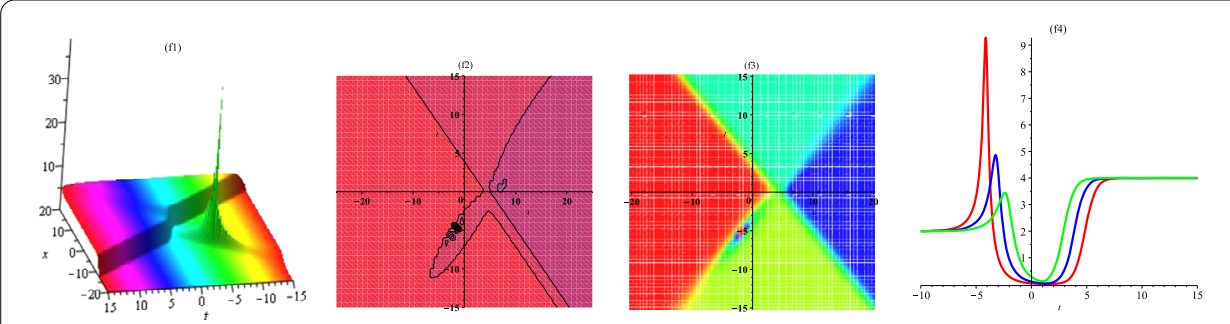

Figure 4 Diagram of 1-lump (2.17) by taking $a=1.5, b=1, k_{1}=1, k_{2}=2, \alpha=1, \epsilon=-1, \tau=2, \eta_{10}=\eta_{20}=0$, $R=2, y=-2$ at space (red $x=-1$, blue $x=0$, and green $x=1$ )
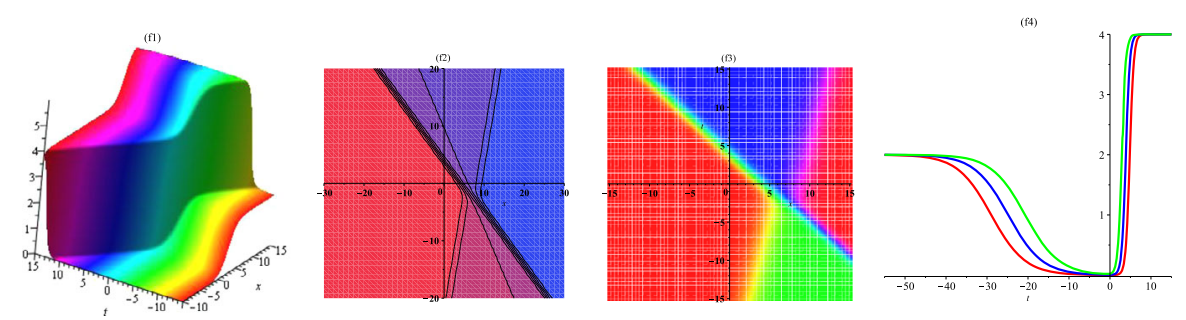

Figure 5 Diagram of 1-lump (2.17) by taking $k_{1}=1, k_{2}=2, \alpha=1, \epsilon=-1, \tau=2, \eta_{10}=\eta_{20}=0, R=2, y=-2$ at space (red $x=-1$, blue $x=0$, and green $x=1$ )

including the 3D plot, density plot, and 2D plot when three spaces arise at spaces $x=-1$, $x=0$, and $x=1$.

\subsection{Multiple-lump solutions for Equation (1.1)}

For computing the multiple-lump solutions of equation (1.1) by putting $N=4$ and $M=2$ in (2.9), we have:

$$
\rho_{4}=1+e^{\zeta_{1}}+e^{\zeta_{2}}+e^{\zeta_{1}+\zeta_{2}+F_{12}}+e^{\zeta_{1}+\zeta_{3}+F_{13}}+e^{\zeta_{2}+\zeta_{3}+F_{23}}+e^{\zeta_{1}+\zeta_{2}+\zeta_{3}+F_{12} F_{13} F_{23}}
$$

where

$$
\begin{aligned}
\zeta_{i}= & k_{i}\left(x+p_{i} y-\left[1+\frac{1}{2} a \alpha p_{i}-\frac{1}{4} \epsilon k_{i}^{2} p_{i}^{2}(2 \tau+1)-\frac{1}{6} \epsilon k_{i}^{2}(3 \tau-1)\right] t\right), \quad i=1,2,3, \\
\exp F_{12} & \\
= & \frac{\left(k_{1}-k_{2}\right)\left(2\left(k_{1}-k_{2}\right)\left(2 \tau p_{1} p_{2}+3 \epsilon \tau+p_{1} p_{2}-\epsilon\right)+\left(k_{1} p_{1}^{2}-k_{2} p_{2}^{2}\right)(2 \tau+1)\right)}{\left(k_{1}+k_{2}\right)\left(2\left(k_{1}+k_{2}\right)\left(2 \tau p_{1} p_{2}+3 \epsilon \tau+p_{1} p_{2}-\epsilon\right)+\left(k_{1} p_{1}^{2}+k_{2} p_{2}^{2}\right)(2 \tau+1)\right)}, \\
\exp F_{13} & \frac{\left(k_{1}-k_{3}\right)\left(2\left(k_{1}-k_{3}\right)\left(2 \tau p_{1} p_{3}+3 \epsilon \tau+p_{1} p_{3}-\epsilon\right)+\left(k_{1} p_{1}^{2}-k_{3} p_{3}^{2}\right)(2 \tau+1)\right)}{\left(k_{1}+k_{3}\right)\left(2\left(k_{1}+k_{3}\right)\left(2 \tau p_{1} p_{3}+3 \epsilon \tau+p_{1} p_{3}-\epsilon\right)+\left(k_{1} p_{1}^{2}+k_{3} p_{3}^{2}\right)(2 \tau+1)\right)}, \\
\exp F_{23} & \left(k_{2}-k_{3}\right)\left(2\left(k_{2}-k_{3}\right)\left(2 \tau p_{2} p_{3}+3 \epsilon \tau+p_{1} p_{3}-\epsilon\right)+\left(k_{2} p_{2}^{2}-k_{3} p_{3}^{2}\right)(2 \tau+1)\right) \\
= & \left(k_{2}+k_{3}\right)\left(2\left(k_{2}+k_{3}\right)\left(2 \tau p_{2} p_{3}+3 \epsilon \tau+p_{1} p_{3}-\epsilon\right)+\left(k_{2} p_{2}^{2}+k_{3} p_{3}^{2}\right)(2 \tau+1)\right)
\end{aligned}
$$




$$
\begin{aligned}
\rho_{4}=1 & +e^{\zeta_{1}}+e^{\zeta_{2}} \\
& +\frac{\left(k_{1}-k_{2}\right)\left(2\left(k_{1}-k_{2}\right)\left(2 \tau p_{1} p_{2}+3 \epsilon \tau+p_{1} p_{2}-\epsilon\right)+\left(k_{1} p_{1}{ }^{2}-k_{2} p_{2}{ }^{2}\right)(2 \tau+1)\right)}{\left(k_{1}+k_{2}\right)\left(2\left(k_{1}+k_{2}\right)\left(2 \tau p_{1} p_{2}+3 \epsilon \tau+p_{1} p_{2}-\epsilon\right)+\left(k_{1} p_{1}{ }^{2}+k_{2} p_{2}{ }^{2}\right)(2 \tau+1)\right)} e^{\zeta_{1}+\zeta_{2}} \\
& +\frac{\left(k_{1}-k_{3}\right)\left(2\left(k_{1}-k_{3}\right)\left(2 \tau p_{1} p_{3}+3 \epsilon \tau+p_{1} p_{3}-\epsilon\right)+\left(k_{1} p_{1}{ }^{2}-k_{3} p_{3}{ }^{2}\right)(2 \tau+1)\right)}{\left(k_{1}+k_{3}\right)\left(2\left(k_{1}+k_{3}\right)\left(2 \tau p_{1} p_{3}+3 \epsilon \tau+p_{1} p_{3}-\epsilon\right)+\left(k_{1} p_{1}{ }^{2}+k_{3} p_{3}{ }^{2}\right)(2 \tau+1)\right)} e^{\zeta_{1}+\zeta_{3}} \\
& +\frac{\left(k_{2}-k_{3}\right)\left(2\left(k_{2}-k_{3}\right)\left(2 \tau p_{2} p_{3}+3 \epsilon \tau+p_{1} p_{3}-\epsilon\right)+\left(k_{2} p_{2}{ }^{2}-k_{3} p_{3}{ }^{2}\right)(2 \tau+1)\right)}{\left(k_{2}+k_{3}\right)\left(2\left(k_{2}+k_{3}\right)\left(2 \tau p_{2} p_{3}+3 \epsilon \tau+p_{1} p_{3}-\epsilon\right)+\left(k_{2} p_{2}{ }^{2}+k_{3} p_{3}{ }^{2}\right)(2 \tau+1)\right)} e^{\zeta_{2}+\zeta_{3}} \\
& +\exp F_{12} \exp F_{13} \exp F_{23} e^{\zeta_{1}+\zeta_{2}+\zeta_{3}},
\end{aligned}
$$

where

$$
\zeta_{j}=x+p_{j} y-\left(1+\frac{1}{2} a \alpha p_{j}\right) t, \quad j=1,2,3
$$

Plugging $\rho_{4}$ into the transmutation $\Psi=R\left(\ln \rho_{4}\right)_{x}$ and taking $p_{1}=a+b i, p_{2}=c+d i, p_{3}=e+f i$ such that $\Re p_{i}>0(i=1,2,3)$, we obtain the 3-lump solution of equation (1.1). In Figs. 6-10 the 3-lump solution is plotted for appropriate values of $p_{1}=a+b i, p_{2}=c+d i, p_{3}=e+f i$. By selecting suitable values of the parameters $\left(p_{1}=a+i b, p_{2}=a-i b, p+3=c+i d\right)$ in Fig. 6, $\left(p_{1}=a+i b, p_{2}=c+i d, p+3=e+i f\right)$ in Fig. 7, $\left(p_{1}=2, p_{2}=3, p+3=4\right)$ in Fig. 8 , $\left(p_{1}=a+i b, p_{2}=3, p+3=c+i d\right)$ in Fig. 9 , and $\left(p_{1}=a+i b, p_{2}=2, p+3=3\right)$ in Fig. 10 the graphical representations of 2-lump (three-soliton) solution are given in Figs. 6-10 containing the 3D plot, density plot, and 2D plot when three spaces arise at spaces $x=-1$, $x=0$, and $x=1$.
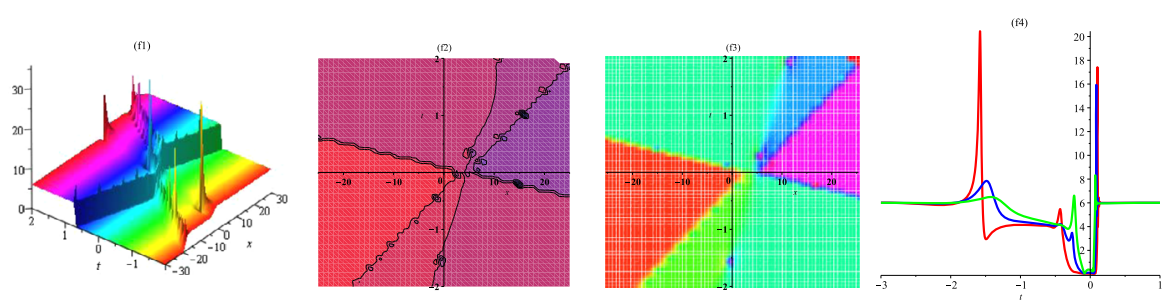

Figure 6 Diagram of 3-lump (2.20) by taking $a=1.5, b=1, c=2, d=3, k_{1}=1, k_{2}=2, k_{3}=3, \alpha=0.1, \epsilon=-1$, $\tau=2, \eta_{10}=\eta_{20}=\eta_{30}=0, R=2, y=-2$ at space $($ red $x=-1$, blue $x=0$, and green $x=1)$
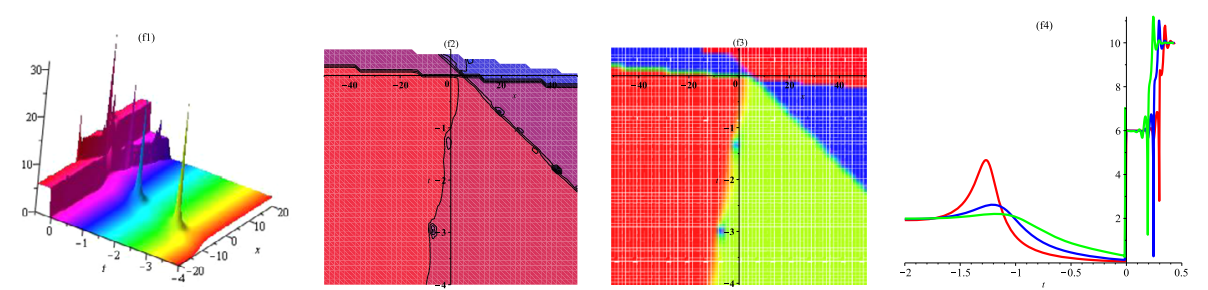

Figure 7 Diagram of 3-lump (2.20) by taking $a=1.5, b=1, c=2, d=3, e=-1, f=5, k_{1}=1, k_{2}=2, k_{3}=3$, $\alpha=0.1, \epsilon=-1, \tau=2, \eta_{10}=\eta_{20}=\eta_{30}=0, R=2, y=-2$ at space (red $x=-1$, blue $x=0$, and green $\left.x=1\right)$ 

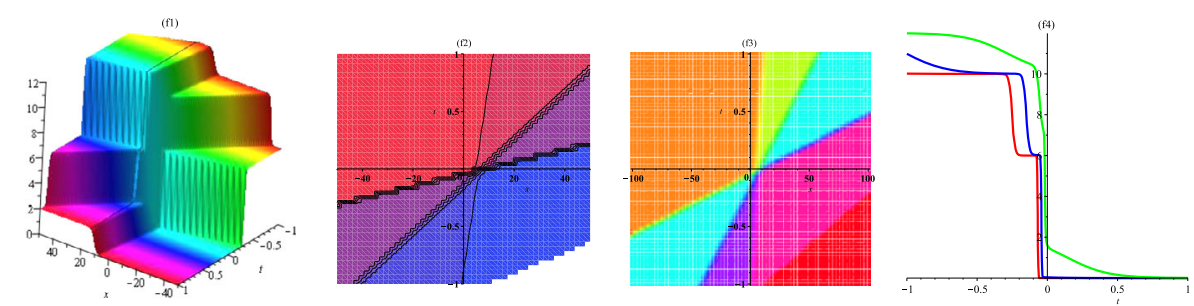

Figure 8 Diagram of 3-lump (2.20) by taking $k_{1}=1, k_{2}=2, k_{3}=3, \alpha=0.1, \epsilon=-1, \tau=2, \eta_{10}=\eta_{20}=\eta_{30}=0$ $R=2, y=-2$ at space (red $x=-1$, blue $x=0$, and green $x=1$ )
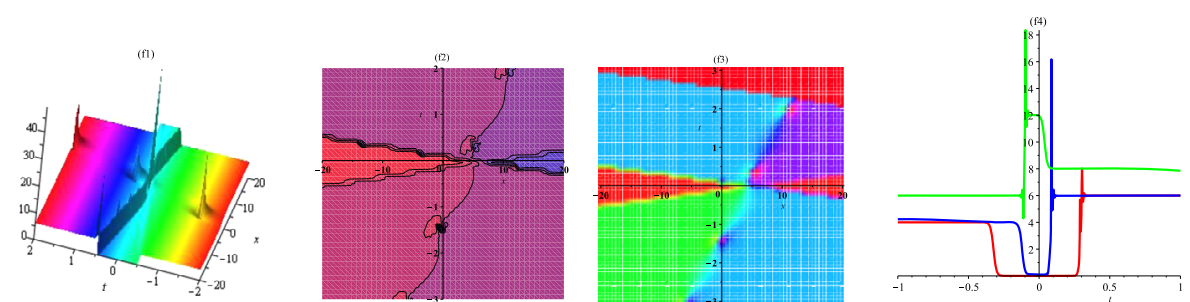

Figure 9 Diagram of 3-lump (2.20) by taking $a=1.5, b=1, c=2, d=3, k_{1}=1, k_{2}=2, k_{3}=3, \alpha=0.1, \epsilon=-1$, $\tau=2, \eta_{10}=\eta_{20}=\eta_{30}=0, R=2, y=-2$, at space $($ red $x=-1$, blue $x=0$, and green $x=1$ )
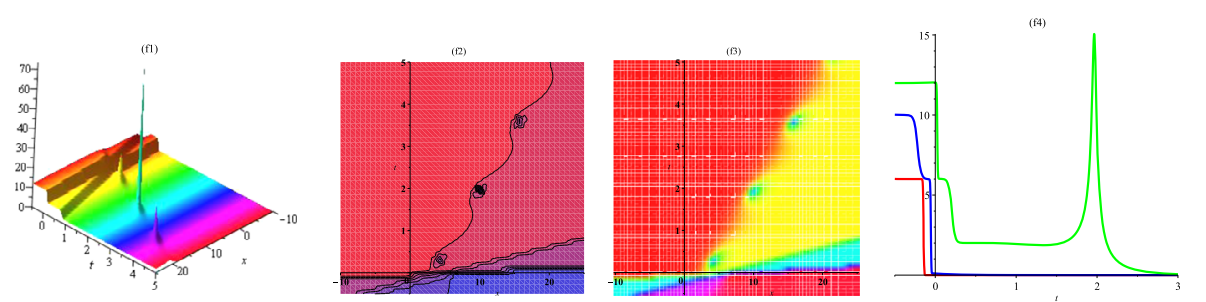

Figure 10 Diagram of 3-lump (2.20) by taking $a=1.5, b=1, c=2, d=3, k_{1}=1, k_{2}=2, k_{3}=3, \alpha=0.1, \epsilon=-1$, $\tau=2, \eta_{10}=\eta_{20}=\eta_{30}=0, R=2, y=-2$, at space (red $x=-1$, blue $x=0$, and green $x=1$ )

\section{Interaction between lumps and stripe solitons of equation (1.1)}

In the following subsections, we further treat diverse solitons.

\subsection{Interaction between 1-lump and 2-stripe soliton of Equation (1.1)}

To treat 1-lump and 1-stripe solitons of equation (1.1), we catch $f$ as a blend of the following functions:

$$
\begin{aligned}
& \rho=\left(\sum_{i=1}^{4} \Omega_{i} x_{i}\right)^{2}+\left(\sum_{i=5}^{8} \Omega_{i} x_{i}\right)^{2}+r_{1}+r_{2} e^{\sum_{i=1}^{4} \Omega_{i} x_{i}}+r_{3} e^{\sum_{i=5}^{8} \Omega_{i} x_{i}}+r_{4} e^{\sum_{i=1}^{8} \Omega_{i} x_{i}} \\
&\left(x_{1}, x_{2}, x_{3}, x_{4}\right)=(x, y, t, 1), \quad\left(x_{5}, x_{6}, x_{7}, x_{8}\right)=(x, y, t, 1) \\
& \Psi=R \frac{\partial}{\partial x} \ln (\rho) \\
&=R \frac{r_{4}\left(\Omega_{1}+\Omega_{5}\right) e^{\sum_{i=1}^{8} \Omega_{i} x_{i}}+2 \Omega_{1} \sum_{i=1}^{4} \Omega_{i} x_{i}+2 \Omega_{5} \sum_{i=5}^{8} \Omega_{i} x_{i}+r_{2} \Omega_{1} e^{\sum_{i=1}^{4} \Omega_{i} x_{i}}+r_{3} \Omega_{5} e^{\sum_{i=5}^{8} \Omega_{i} x_{i}}}{\rho},
\end{aligned}
$$


where $\left(x_{1}, x_{2}, x_{3}\right)=(x, y, t)$, and $\Omega_{i}, i=1, \ldots, 8, r_{1}, r_{2}, r_{3}$ are free parameters to be found later. Plugging (3.1) into Eq. (1.3), collecting the coefficients at the diverse polynomial functions including the functions $e^{\sum_{i=1}^{4} \Omega_{i} x_{i}}, e^{\sum_{i=5}^{8} \Omega_{i} x_{i}}, e^{\sum_{i=1}^{8} \Omega_{i} x_{i}}$ and their products, and solving the obtained algebraic system containing 26 equations, we obtain the following solutions:

Set I:

$$
\left\{\begin{array}{l}
\tau=-\frac{1}{6} \frac{6 \Omega_{2}^{2} \sqrt{2}+2 \epsilon \Omega_{5}^{2}-9 \Omega_{2}^{2}}{2 \Omega_{2}^{2} \sqrt{2}-\epsilon \Omega_{5}^{2}-3 \Omega_{2}^{2}}, \quad \Omega_{1}=(1+\sqrt{2}) \Omega_{5}, \quad \Omega_{2}=\Omega_{2}, \\
\Omega_{3}=-\frac{1}{2} a \alpha \Omega_{2}-(1+\sqrt{2}) \Omega_{5}, \quad \Omega_{4}=0, \quad \Omega_{5}=\Omega_{5}, \\
\Omega_{6}=\Omega_{2}(\sqrt{2}-1), \quad \Omega_{7}=-\frac{1}{2}(\sqrt{2}-1)\left(a \alpha \Omega_{2}+2(\sqrt{2}+1) \Omega_{5}\right), \quad \Omega_{8}=0, \\
r_{1}=r_{1}, \quad r_{2}=r_{2}, \quad r_{3}=0, \quad r_{4}=r_{4},
\end{array}\right.
$$

which should satisfy the condition

$$
(2 \sqrt{2}-3) \Omega_{2}^{2}-\epsilon \Omega_{5}^{2} \neq 0 .
$$

Plugging (3.3) into (3.2), we achieve te following interactive wave solution of Eq. (1.1):

$$
\begin{aligned}
& \Psi_{1}=R \frac{r_{4}(2+\sqrt{2}) \Omega_{5} e^{\sum_{i=1, i \neq 4}^{7} \Omega_{i} x_{i}}+2 \Omega_{1} \sum_{i=1}^{3} \Omega_{i} x_{i}+2 \Omega_{5} \sum_{i=5}^{7} \Omega_{i} x_{i}+r_{2} \Omega_{1} e^{\sum_{i=1}^{3} \Omega_{i} x_{i}}}{\rho}, \\
& \rho=\left(\sum_{i=1}^{3} \Omega_{i} x_{i}\right)^{2}+\left(\sum_{i=5}^{7} \Omega_{i} x_{i}\right)^{2}+r_{1}+r_{2} e^{\sum_{i=1}^{3} \Omega_{i} x_{i}}+r_{4} e^{\sum_{i=1}^{7} \Omega_{i} x_{i}} .
\end{aligned}
$$

Moreover, by selecting suitable values of the parameters the graphic representation of periodic wave solution is presented in Fig. 11, including the 3D plot, density plot, and 2D plot when three spaces arise at spaces $x=-1, x=0$, and $x=1$.

Set II:

$$
\left\{\begin{array}{l}
\tau=\frac{1}{6} \frac{2 \epsilon \Omega_{1}{ }^{2}-3 \Omega_{2}{ }^{2}}{\epsilon \Omega_{1}{ }^{2}+\Omega_{2}{ }^{2}}, \quad \Omega_{1}=\Omega_{1}, \quad \Omega_{2}=\Omega_{2}, \\
\Omega_{3}=-\frac{1}{2} a \alpha \Omega_{2}-\Omega_{1}, \quad \Omega_{4}=\Omega_{4}, \quad \Omega_{5}=\Omega_{5}, \quad \Omega_{6}=\frac{\Omega_{2} \Omega_{5}}{\Omega_{1}}, \\
\Omega_{7}=-\frac{\left(a \alpha \Omega_{2}+2 \Omega_{1}\right) \Omega_{5}}{2 \Omega_{1}}, \quad \Omega_{8}=\Omega_{8}, \\
r_{1}=r_{1}, \quad r_{2}=r_{2}, \quad r_{3}=r_{3}, \quad r_{4}=r_{4},
\end{array}\right.
$$

which should satisfy the condition

$$
\epsilon \Omega_{1}^{2}+\Omega_{2}^{2} \neq 0
$$

Plugging (3.6) into (3.2), we achieve the following interactive wave solution of Eq. (1.1):

$$
\begin{aligned}
& \Psi_{2}=R \frac{r_{4}\left(\Omega_{1}+\Omega_{5}\right) e^{\sum_{i=1}^{8} \Omega_{i} x_{i}}+2 \Omega_{1} \sum_{i=1}^{4} \Omega_{i} x_{i}+2 \Omega_{5} \sum_{i=5}^{8} \Omega_{i} x_{i}+r_{2} \Omega_{1} e^{\sum_{i=1}^{4} \Omega_{i} x_{i}}+r_{3} \Omega_{5} e^{\sum_{i=5}^{8} \Omega_{i} x_{i}}}{\rho}, \\
& \rho=\left(\sum_{i=1}^{4} \Omega_{i} x_{i}\right)^{2}+\left(\sum_{i=5}^{8} \Omega_{i} x_{i}\right)^{2}+r_{1}+r_{2} e^{\sum_{i=1}^{4} \Omega_{i} x_{i}}+r_{3} e^{\sum_{i=5}^{8} \Omega_{i} x_{i}}+r_{4} e^{\sum_{i=1}^{8} \Omega_{i} x_{i}}
\end{aligned}
$$




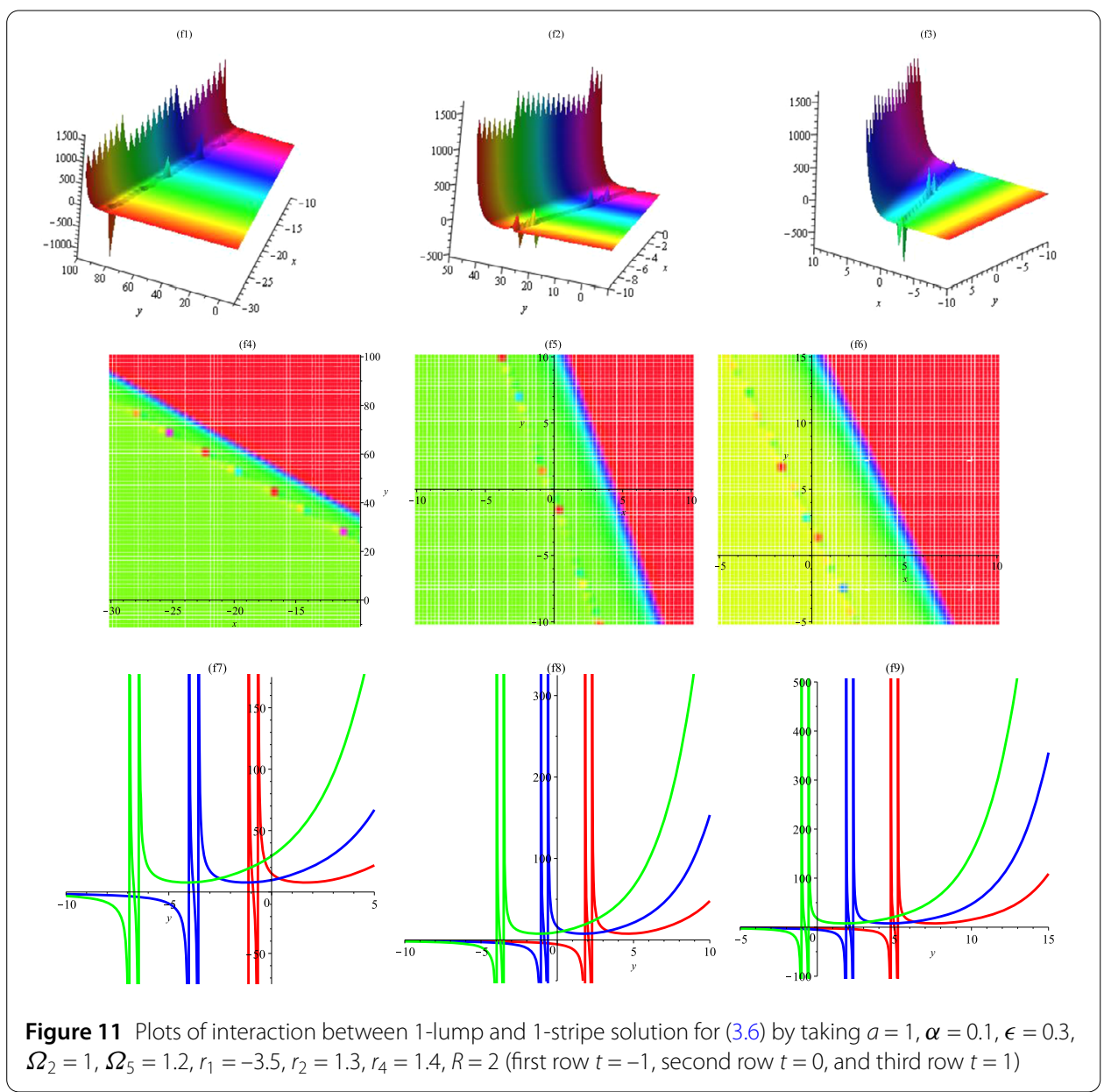

Likewise, by selecting suitable values of the parameters the graphic representation of periodic wave solution is presented in Fig. 12, containing the 3D plot, density plot, and 2D plot when three spaces arise at spaces $x=-1, x=0$, and $x=1$.

Set III:

$$
\left\{\begin{array}{l}
\tau=-\frac{1}{6} \frac{3 \Omega_{2}^{2} \sqrt{17}-8 \epsilon \Omega_{5}^{2}+9 \Omega_{2}^{2}}{\Omega_{2}^{2} \sqrt{17}+4 \epsilon \Omega_{5}^{2}+3 \Omega_{2}^{2}}, \quad \Omega_{1}=\frac{1}{2} \sqrt{-6+2 \sqrt{17}} \Omega_{5}, \\
\Omega_{2}=\Omega_{2}, \quad \Omega_{3}=-\frac{1}{2} a \alpha \Omega_{2}-\frac{1}{2} \sqrt{-6+2 \sqrt{17}} \Omega_{5}, \\
\Omega_{4}=0, \quad \Omega_{5}=\Omega_{5}, \quad \Omega_{6}=\frac{1}{8} \sqrt{-6+2 \sqrt{17}}(\sqrt{17}+3) \Omega_{2}, \\
\Omega_{7}=-\frac{1}{16} \sqrt{-6+2 \sqrt{17}}(\sqrt{17}+3)\left(\sqrt{-6+2 \sqrt{17}} \Omega_{5}+a \alpha \Omega_{2}\right), \\
\Omega_{8}=0, \quad r_{1}=r_{1}, \quad r_{2}=r_{2}, \\
r_{3}=\frac{\left(912 r_{1}+10412+19 \sqrt{17}\left(11 r_{1}+124\right)+19 \sqrt{-6+2 \sqrt{17}}(2 \sqrt{17}+7)\left(5 \sqrt{17}+25+4 r_{1}\right)\right) r_{4}}{361 r_{2}}, \quad r_{4}=r_{4},
\end{array}\right.
$$

which should satisfy the condition

$$
(\sqrt{17}+3) \Omega_{2}^{2}+4 \epsilon \Omega_{5}^{2} \neq 0
$$




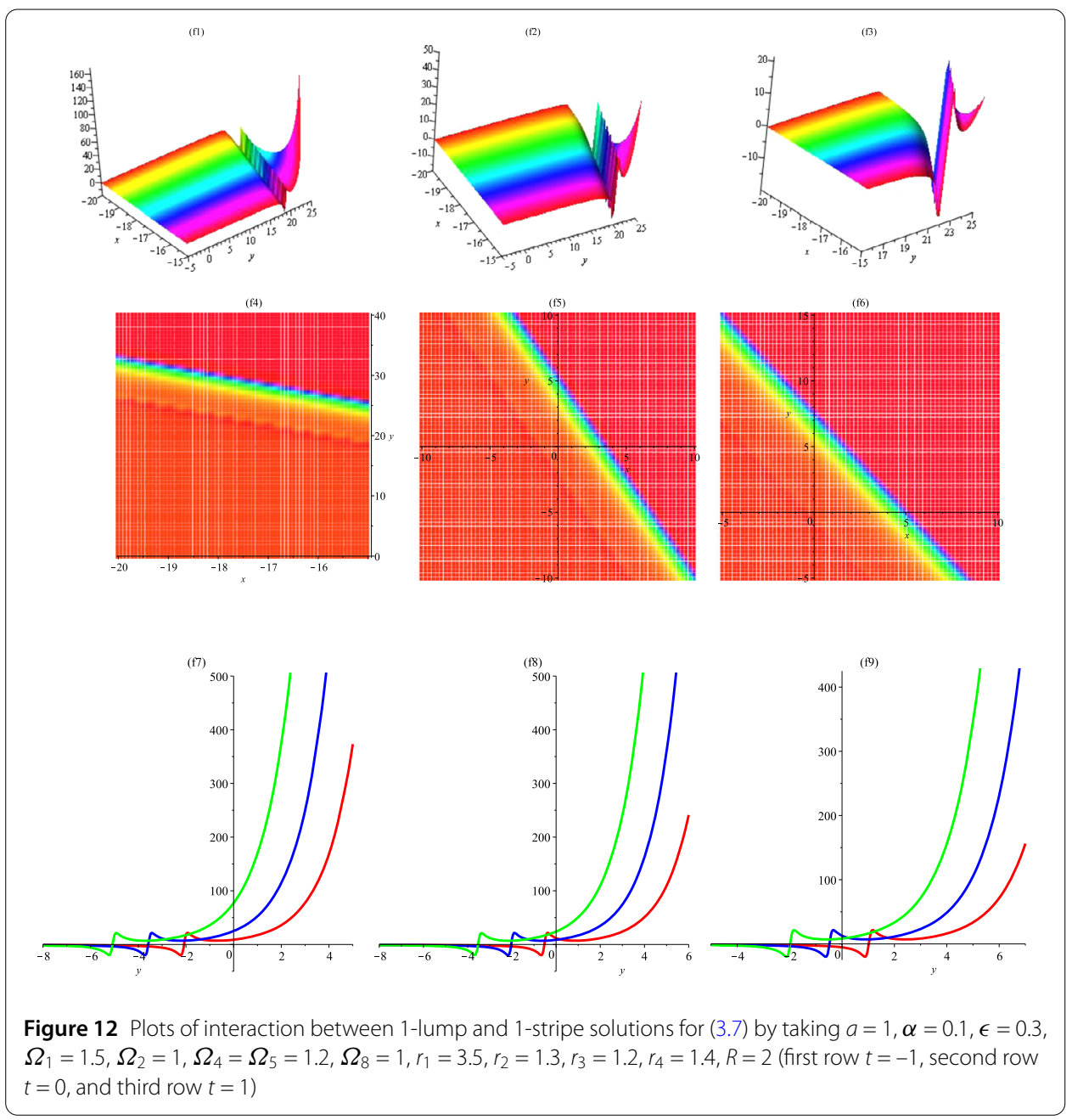

Plugging (3.9) into (3.2), we achieve the following interactive wave solution of Eq. (1.1):

$$
\begin{aligned}
& \Psi_{3}=R \frac{r_{4}\left(\Omega_{1}+\Omega_{5}\right) e^{\sum_{i=1,74}^{7} \Omega_{i} x_{i}}+2 \Omega_{1} \sum_{i=1}^{3} \Omega_{i} x_{i}+2 \Omega_{5} \sum_{i=5}^{7} \Omega_{i} x_{i}+r_{2} \Omega_{1} e^{\sum_{i=1}^{3} \Omega_{i} x_{i}}+r_{3} \Omega_{5} e^{\sum_{i=5}^{7} \Omega_{i} x_{i}}}{\rho}, \\
& \rho=\left(\sum_{i=1}^{3} \Omega_{i} x_{i}\right)^{2}+\left(\sum_{i=5}^{7} \Omega_{i} x_{i}\right)^{2}+r_{1}+r_{2} e^{\sum_{i=1}^{3} \Omega_{i} x_{i}}+r_{3} e^{\sum_{i=5}^{7} \Omega_{i} x_{i}}+r_{4} e^{\sum_{i=1,74}^{7} \Omega_{i} x_{i}} \cdot
\end{aligned}
$$

Remark 3.1 By selecting suitable values of the parameters, the graphical representations of interaction solutions are presented in Figs. 11-12. These figures suggest that there are 1-lump and 2-stripe solitons, the energy of the 1-lump is more robust than that of the 2stripe soliton; as $t \rightarrow 0$, the 1-lump commences to be swallowed by the 2 -stripe soliton gradually, its energy commences to move from one place to another into the 2-stripe soliton progressively, until it is swallowed by the stripe soliton completely. These two types of solutions move into one soliton and continue to spread. 


\subsection{Interaction between 2-lump and 1-stripe solitons of equation (1.1)}

To search treatment between 2-lump and 1-stripe solitons of equation (1.1), we catch $f$ as a blend of the following functions:

$$
\begin{aligned}
& \rho=\left(\sum_{i=1}^{4} \Omega_{i} x_{i}\right)^{4}+2\left(\sum_{i=1}^{4} \Omega_{i} x_{i}\right)^{2}+r+k e^{\sum_{i=5}^{8} \Omega_{i} x_{i}}, \\
& \left(x_{1,5}, x_{2,6}, x_{3,7}, x_{4,8}\right)=(x, y, t, 1), \\
& \Psi=R \frac{\partial}{\partial x} \ln (\rho)=R \frac{4 \Omega_{1}\left(\sum_{i=1}^{4} \Omega_{i} x_{i}\right)^{3}+4 \Omega_{1} \sum_{i=1}^{4} \Omega_{i} x_{i}+k \Omega_{5} e^{\sum_{i=5}^{8} \Omega_{i} x_{i}}}{\rho},
\end{aligned}
$$

where $\Omega_{i}, i=1, \ldots, 4, r, k$ are free elements to be defined later. Plugging (3.12) into Eq. (1.3), collecting the coefficients at the diverse polynomial functions including $e^{\sum_{i=5}^{8} \Omega_{i} x_{i}}$ and their products, and solving the obtained algebraic system of 11 equations, we get the following solutions:

Set I:

$$
\left\{\begin{array}{l}
\Omega_{1}=\Omega_{1}, \quad \Omega_{2}=-\frac{2 \Omega_{1}(3 \tau-1)}{\sqrt{-(12 \tau+6)(3 \tau-1)}}, \\
\Omega_{3}=-\frac{1}{6} \frac{\Omega_{1} \sqrt{-(12 \tau+6)(3 \tau-1)} a \alpha}{2 \tau+1}-\Omega_{1}, \quad \Omega_{4}=\Omega_{4}, \\
\Omega_{5}=\Omega_{5}, \quad \Omega_{6}=\frac{1}{3} \frac{\sqrt{-(12 \tau+6)(3 \tau-1)} \Omega_{5}}{2 \tau+1}, \\
\Omega_{7}=-\frac{1}{6} \frac{a \alpha \sqrt{-(12 \tau+6)(3 \tau-1)} \Omega_{5}}{2 \tau+1}-\Omega_{5}, \quad \Omega_{8}=\Omega_{8} .
\end{array}\right.
$$

To ensure the positivity of $\rho$, we need the following determinant condition:

$$
(12 \tau+6)(3 \tau-1)<0, \quad \tau \neq-\frac{1}{2}
$$

Plugging (3.14) into (3.13), we get the following interactive wave solution of Eq. (1.1):

$$
\Psi_{1}=R \frac{4 \Omega_{1}\left(\sum_{i=1}^{4} \Omega_{i} x_{i}\right)^{3}+4 \Omega_{1} \sum_{i=1}^{4} \Omega_{i} x_{i}+k \Omega_{5} e^{\sum_{i=5}^{8} \Omega_{i} x_{i}}}{\rho},
$$

where

$$
\rho=\left(\sum_{i=1}^{4} \Omega_{i} x_{i}\right)^{4}+2\left(\sum_{i=1}^{4} \Omega_{i} x_{i}\right)^{2}+r+k e^{\sum_{i=5}^{8} \Omega_{i} x_{i}}
$$

Set II:

$$
\left\{\begin{array}{l}
\Omega_{1}=\frac{3}{10} \frac{\Omega_{4} \sqrt{-(12 \tau+6)(3 \tau-1)} \Omega_{6}}{3 \tau-1}, \quad \Omega_{2}=\frac{3}{5} \Omega_{4} \Omega_{6}, \\
\Omega_{3}=-\frac{3}{10} \Omega_{4} \Omega_{6}\left(a \alpha+\frac{\sqrt{-(12 \tau+6)(3 \tau-1)}}{3 \tau-1}\right), \quad \Omega_{4}=\Omega_{4}, \\
\Omega_{5}=\frac{1}{2} \frac{\sqrt{-(12 \tau+6)(3 \tau-1)} \Omega_{6}}{3 \tau-1}, \quad \Omega_{6}=\Omega_{6}, \\
\Omega_{7}=-\frac{1}{2} \Omega_{6}\left(a \alpha+\frac{\sqrt{-(12 \tau+6)(3 \tau-1)}}{3 \tau-1}\right), \quad \Omega_{8}=\Omega_{8} .
\end{array}\right.
$$

To ensure the positivity of $\rho$, we need the following determinant condition:

$$
(12 \tau+6)(3 \tau-1)<0, \quad \tau \neq \frac{1}{3}
$$




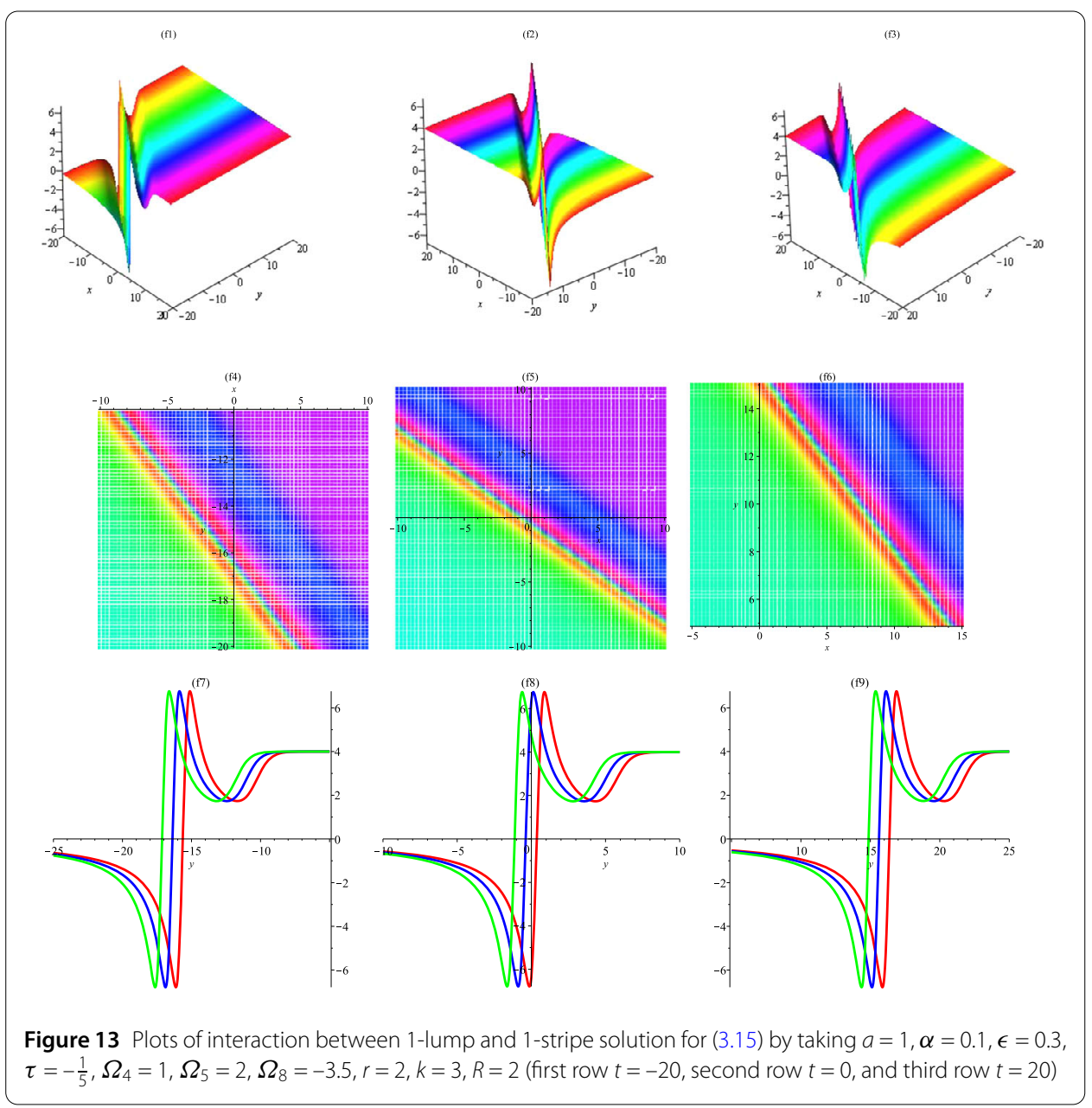

Plugging (3.16) into (3.13), we get the following interactive wave solution of Eq. (1.1):

$$
\Psi_{2}=R \frac{\frac{6}{5} \frac{\Omega_{4} \sqrt{-(12 \tau+6)(3 \tau-1)} \Omega_{6}}{3 \tau-1}\left(\sum_{i=1}^{4} \Omega_{i} x_{i}\right)^{3}+\frac{6}{5} \frac{\Omega_{4} \sqrt{-(12 \tau+6)(3 \tau-1)} \Omega_{6}}{3 \tau-1} \sum_{i=1}^{4} \Omega_{i} x_{i}+\frac{k}{2} \frac{\sqrt{-(12 \tau+6)(3 \tau-1)} \Omega_{6}}{3 \tau-1} e^{\sum_{i=5}^{8} \Omega_{i} x_{i}}}{\rho},
$$

where

$$
\rho=\left(\sum_{i=1}^{4} \Omega_{i} x_{i}\right)^{4}+2\left(\sum_{i=1}^{4} \Omega_{i} x_{i}\right)^{2}+r+k e^{\sum_{i=5}^{8} \Omega_{i} x_{i}} .
$$

Remark 3.2 By selecting suitable values of the parameters, the graphical representations of interaction solutions are presented in Figs. 13 and 14. By selecting suitable values of the parameters, the graphical representation of interaction between 2-lump and 1-stripe solitons is presented in Figs. 13 and 14. These figures show that there are 2-lump and a 1 -stripe solitons; as $t \rightarrow 0$, 2-lump commences to be swallowed by 2 -stripe soliton gradually, its energy commences to move from one place to another into the 1-stripe soliton progressively, until it is swallowed by the 1-stripe soliton generally, and these two types of solutions move into one resonance soliton and continue to spread. 


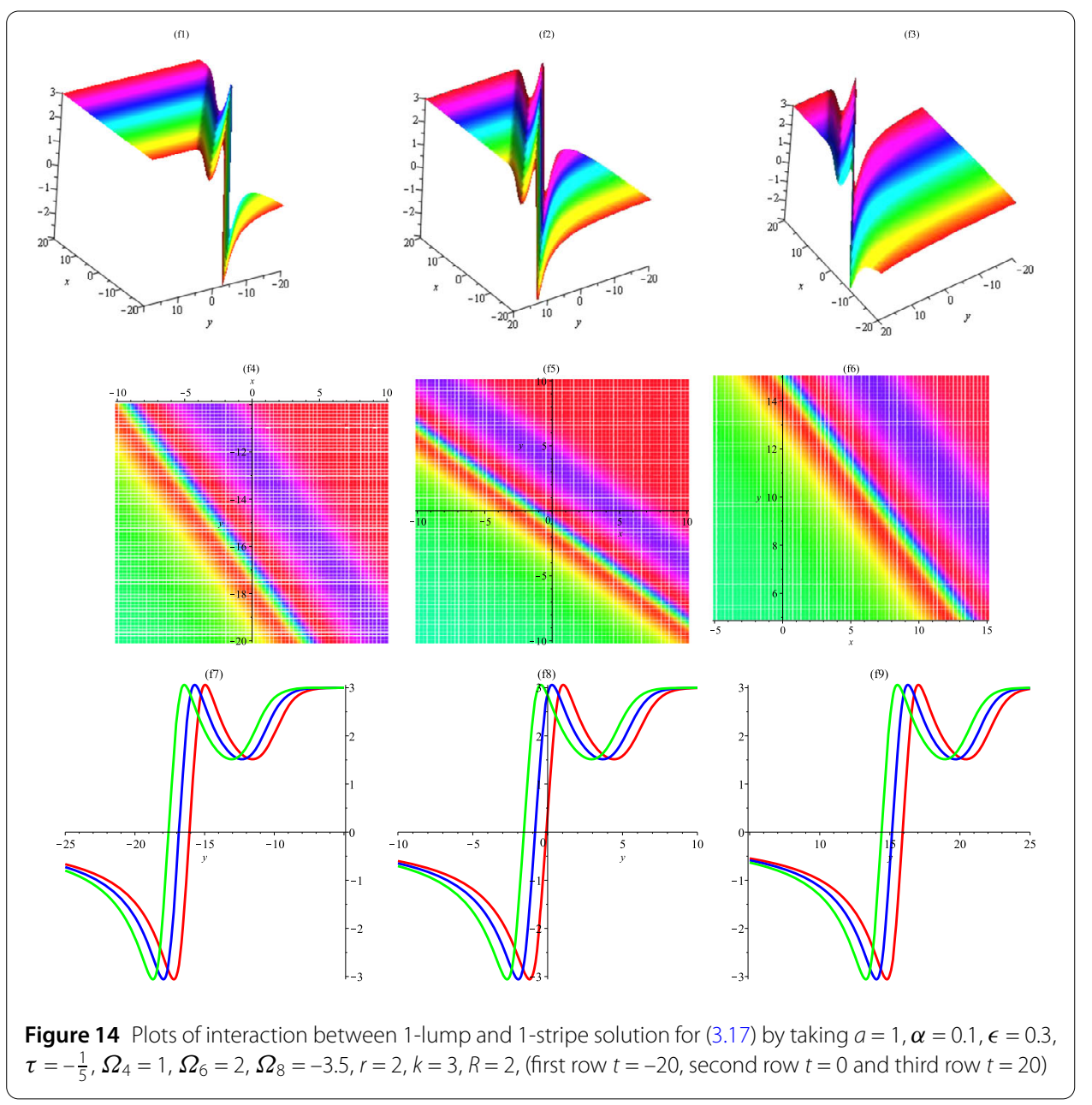

\section{Conclusions}

We employed the Hirota bilinear method, along with some Hirota derivatives and the Bell polynomial theories of soliton equations, to find abundantly many exact lumps and interaction lumps with two types of typical local excitations, which occurred between a lump and a stripe soliton of soliton solutions to third-order evaluation equation. We investigated $M$ lump solutions and made different types of graphs, including the contour, density, and three- and two dimensional plots. We also obtained an interaction between 1-lump and two-stripe solutions and an interaction between 2-lump and one-stripe solutions and found more general rational exact soliton wave solutions of the third-order evaluation equation. This approach has been successfully applied to obtain some real rational soliton wave solutions to third-order evaluation equation with constant coefficients. We proved a theorem for the considered problem. We also obtained existence criteria of these solitons in the unidirectional propagation of long waves over shallow water. The attained solutions are in broad-ranging form, and the definite values of the included parameters of the attained solutions yield the soliton solutions and are helpful in analyzing the water waves mechanics, the quantum mechanics, the water waves in gravitational force, the signal processing waves, the optical fibers, and so on. This paper showed that the Hirota bilinear method, combined with Hirota derivatives, gives a unified approach to constructing the exact rational lump soliton wave solutions to many nonlinear partial differential 
equations. Our results allowed us to understand the dynamics of nonlinear propagation in fluid mechanics, plasma, and so on. Moreover, the established results have shown that the Hirota bilinear method is general, straightforward, and powerful and helped us to examine traveling wave solutions of NLPDEs.

\section{Acknowledgements}

Not applicable.

\section{Funding}

This work was not supported by any specific funding.

\section{Availability of data and materials}

The data sets supporting the conclusions of this papere are included within the papere and its additional file.

\section{Competing interests}

The authors declare they have no competing interest.

\section{Conflict of interests}

The authors declare that they have no conflicts of interest.

\section{Authors' contributions}

$\mathrm{JM}$ and OAI made the numerical simulations and wrote the paper. JM and AA provided the Hirota method and applying it solved the problem in Sect. 2. OAI and SAM provided Sect. 3.1 in the paper. Also, Sect. 3.2 has been provided by AA and SAM. All authors read and approved the final manuscript.

\section{Author details}

${ }^{1}$ Department of Mathematics, Faculty of Education, Erciyes University, Kayseri, Turkey. ${ }^{2}$ Department of Applied Mathematics, Faculty of Mathematical Sciences, University of Tabriz, Tabriz, Iran. ${ }^{3}$ Department of Mechanical Engineering Urmia University of Technology, Urmia, Iran. ${ }^{4}$ Department of Mechanical Engineering, College of Engineering, University of Zakho, Zakho, Iraq. ${ }^{5}$ Department of Mathematics, College of Basic Education, University of Duhok, Duhok, Iraq.

\section{Publisher's Note}

Springer Nature remains neutral with regard to jurisdictional claims in published maps and institutional affiliations.

Received: 24 October 2019 Accepted: 28 April 2020 Published online: 12 May 2020

\section{References}

1. Zennir, K., Alodhaibi, S.S.: A novel decay rate for a coupled system of nonlinear viscoelastic wave equations. Mathematics 8(2), 203 (2020)

2. Manafian, J., Heidari, S.: Periodic and singular kink solutions of the Hamiltonian amplitude equation. Adv. Math. Models Appl. 4(2), 134-149 (2019)

3. Kudryashov, N.A., Sinelshchikov, D.I.: Extended models of non-linear waves in liquid with gas bubbles. Int. J. Non-Linear Mech. 63, 31-38 (2014)

4. Abdou, M., Hendi, A., Alanzi, H.K.: New exact solutions of KdV equation in an elastic tube filled with a variable viscosity fluid. Stud. Nonlinear Sci. 3, 62-68 (2012)

5. Johnson, R.: A non-linear equation incorporating damping and dispersion. J. Fluid Mech. 42, 49-60 (1970)

6. Leblond, H., Mihalache, D.: Ultrashort light bullets described by the two-dimensional sine-Gordon equation. Phys. Rev. A 81, 063815 (2010)

7. Bilyay, E., Ozbahceci, B., Yalciner, A.: Extreme waves at Filyos, Southern Black Sea. Nat. Hazards Earth Syst. Sci. 11, 659-666 (2011)

8. Manafian, J. Lakestani, M: Abundant soliton solutions for the Kundu-Eckhaus equation via $\tan (\phi / 2)$-expansion method. Optik 127, 5543-5551 (2016)

9. Manafian, J.: On the complex structures of the Biswas-Milovic equation for power, parabolic and dual parabolic law nonlinearities. Eur. Phys. J. Plus 130, 1-20 (2015)

10. Baskonus, H.M., Bulut, H.: Exponential prototype structures for $(2+1)$-dimensional Boiti-Leon-Pempinelli systems in mathematical physics. Waves Random Complex Media 26, 201-208 (2016)

11. Manafian, J., Foroutan, M.R., Guzali, A.: Applications of the ETEM for obtaining optical soliton solutions for the Lakshmanan-Porsezian-Daniel model. Eur. Phys. J. Plus 132, 494 (2017)

12. Zhou, Q., Ekici, M., Sonmezoglu, A., Manafian, J., Khaleghizadeh, S., Mirzazadeh, M.: Exact solitary wave solutions to the generalized Fisher equation. Optik 127, 12085-12092 (2016)

13. Manafian, J.: Optical soliton solutions for Schrödinger type nonlinear evolution equations by the $\tan (\phi / 2)$-expansion method. Optik 127, 4222-4245 (2016)

14. Manafian, J., Lakestani, M.: Dispersive dark optical soliton with Tzitzéica type nonlinear evolution equations arising in nonlinear optics. Opt. Quantum Electron. 48, 1-32 (2016)

15. Seyedi, S.H., Saray, B.N., Chamkha, A.J.: Heat and mass transfer investigation of MHD Eyring-Powell flow in a stretching channel with chemical reactions. Phys. A, Stat. Mech. Appl. 544, 124109 (2020)

16. Sindi, C.T., Manafian, J.: Wave solutions for variants of the KdV-Burger and the $K(n, n)$-Burger equations by the generalized $G^{\prime} / G$-expansion method. Math. Methods Appl. Sci. 87, 1-14 (2016) 
17. Satsuma, J., Ablowitz, M.J.: Two-dimensional lumps in nonlinear dispersive systems. J. Math. Phys. 20(7), 1496-1503 (1979)

18. Ma, W.X., Zhou, Y., Dougherty, R.: Lump-type solutions to nonlinear differential equations derived from generalized bilinear equations. Int. J. Mod. Phys. B 30(28n29), 1640018 (2016)

19. Lü, J., Bilige, S., Gao, X., Bai, Y., Zhang, R.: Abundant lump solution and interaction phenomenon to Kadomtsev-Petviashvili-Benjamin-Bona-Mahony equation. J. Appl. Math. Phys. 6, 1733-1747 (2018)

20. Wang, C.J.: Spatiotemporal deformation of lump solution to $(2+1)$-dimensional KdV equation. Nonlinear Dyn. 84 697-702 (2016)

21. Foroutan, M.R., Manafian, J., Ranjbaran, A.: Lump solution and its interaction to ( $3+1)$-D potential-YTSF equation. Nonlinear Dyn. 92(4), 2077-2092 (2018)

22. Tang, Y.N., Tao, S.Q., Guan, Q.: Lump solitons and the interaction phenomena of them for two classes of nonlinear evolution equations. Comput. Math. Appl. 72, 2334-2342 (2016)

23. Ma, W.X.: Lump solutions to the Kadomtsev-Petviashvili equation. Phys. Lett. A 379, 1975-1978 (2015)

24. Yang, J.Y., Ma, W.X.: Lump solutions to the BKP equation by symbolic computation. Int. J. Mod. Phys. B 30, 1640028 (2016). https://doi.org/10.1142/S0217979216400282

25. Ma, W.X., Qin, Z.Y., LV, X.: Lump solutions to dimensionally reduced p-gKP and p-gBKP equations. Nonlinear Dyn. 84 923-931 (2016). https://doi.org/10.1007/s11071-015-2539-6

26. Wang, C.J.: Spatiotemporal deformation of lump solution to $(2+1)$-dimensional KdV equation. Nonlinear Dyn. 84 697-702 (2016)

27. Manafian, J., Mohammadi Ivatlo, B., Abapour, M.: Lump-type solutions and interaction phenomenon to the $(2+1)$-dimensional breaking soliton equation. Appl. Math. Comput. 13,13-41 (2019)

28. Ilhan, O.A., Manafian, J., Shahriari, M.: Lump wave solutions and the interaction phenomenon for a variable-coefficient Kadomtsev-Petviashvili equation. Comput. Math. Appl. 78(8), 2429-2448 (2019)

29. Ilhan, O.A., Manafian, J.: Periodic type and periodic cross-kink wave solutions to the $(2+1)$-dimensional breaking soliton equation arising in fluid dynamics. Mod. Phys. Lett. B 33(23), 1950277 (2019)

30. Huang, L.L., Chen, Y.: Lump solutions and interaction phenomenon for $(2+1)$-dimensional Sawada-Kotera equation. Commun. Theor. Phys. 67(5), 473-478 (2017)

31. Lu, J.Q., Bilige, S.D.: Lump solutions of a (2 + 1)-dimensional bSK equation. Nonlinear Dyn. 90, 2119-2124 (2017)

32. Manafian, J., Lakestani, M.: Lump-type solutions and interaction phenomenon to the bidirectional Sawada-Kotera equation. Pramana 92, 41 (2019)

33. Lü, J., Bilige, S., Chaolu, T.: The study of lump solution and interaction phenomenon to $(2+1)$-dimensional generalized fifth-order KdV equation. Nonlinear Dyn. 91, 1669-1676 (2018). https://doi.org/10.1007/s1 1071-017-3972-5

34. Wang, C.J., Dai, Z.D., Liu, C.F.: Interaction between kink solitary wave and rogue wave for $(2+1)$-dimensional Burgers equation. Mediterr. J. Math. 13, 1087-1098 (2016)

35. Zhang, Y., Dong, H.H., Zhang, X.E., et al.: Rational solutions and lump solutions to the generalized $(3+1)$-dimensional shallow water-like equation. Comput. Math. Appl. 73, 246-252 (2017)

36. Wang, J., An, H.L., Li, B.: Non-traveling lump solutions and mixed lump-kink solutions to (2+1)-dimensional variable-coefficient Caudrey-Dodd-Gibbon-Kotera-Sawada equation. Mod. Phys. Lett. B 33(22), 1950262 (2019)

37. Manafian, J.: Novel solitary wave solutions for the $(3+1)$-dimensional extended Jimbo-Miwa equations. Comput. Math. Appl. 76(5), 1246-1260 (2018)

38. Dai, Z.D., Liu, J., Zeng, X.P., Liu, Z.J.: Periodic kink-wave and kinky periodic-wave solutions for the Jimbo-Miwa equation. Phys. Lett. A 372, 5984-5986 (2008)

39. Geng, X.G., Ma, Y.L.: N-Soliton solution and its ronskian form of a $(3+1)$-dimensional nonlinear evolution equation. Phys. Lett. A 369(4), 285-289 (2007)

40. Ilhan, O.A., Manafian, J.: Periodic type and periodic cross-kink wave solutions to the $(2+1)$-dimensional breaking soliton equation arising in fluid dynamics. Mod. Phys. Lett. B 33(23), 1950277 (2019). https://doi.org/10.1142/S0217984919502774

41. Dehghan, M., Manafian, J., Saadatmandi, A.: Application of the Exp-function method for solving a partial differential equation arising in biology and population genetics. Int. J. Numer. Methods Heat Fluid Flow 21, 736-753 (2011)

42. Ma, W.X., Zhu, Z:: Solving the (3+1)-dimensional generalized KP and BKP equations by the multiple exp-function algorithm. Appl. Math. Comput. 218, 11871-11879 (2012)

43. Dehghan, M., Manafian, J.: The solution of the variable coefficients fourth-order parabolic partial differential equations by homotopy perturbation method. Z. Naturforsch. A 64a, 420-430 (2009)

44. Ramani, A.: Inverse scattering, ordinary differential equations of Painlev́e type and Hirota's bilinear formalism. Ann. N.Y. Acad. Sci. 373, 54-67 (1981)

45. Ma, W.X.: Lump solutions to the Kadomtsev-Petviashvili equation. Phys. Lett. A 379, 1975-1978 (2015)

46. Gao, X.Y., Guo, Y.J., Shan, W.R.: Water-wave symbolic computation for the Earth, Enceladus and Titan: the higher-order Boussinesq-Burgers system, auto- and non-auto-Backlund transformations. Appl. Math. Lett. 104, 106170 (2020)

47. Chen, S.S., Tian, B., Liu, L., Yuan, Y.Q., Zhang, C.R.: Conservation laws, binary Darboux transformations and solitons for a higher-order nonlinear Schrödinger system. Chaos Solitons Fractals 118, 337-346 (2019)

48. Hu, C.C., Tian, B., Wu, X.Y., Yuan, Y.Q., Du, Z.: Mixed lump-kink and rogue wave-kink solutions for a $(3+1)$-dimensional B-type Kadomtsev-Petviashvili equation in fluid mechanics. Eur. Phys. J. Plus 133, 40 (2018)

49. Du, X.X., Tian, B., Wu, X.Y., Yin, H.M., Zhang, C.R.: Lie group analysis, analytic solutions and conservation laws of the $(3+1)$-dimensional Zakharov-Kuznetsov-Burgers equation in a collisionless magnetized electron-positron-ion plasma. Eur. Phys. J. Plus 133, 378 (2018)

50. Zhang, C.R., Tian, B., Wu, X.Y., Yuan, Y.Q., Du, X.X.: Rogue waves and solitons of the coherently-coupled nonlinear Schrödinger equations with the positive coherent coupling. Phys. Scr. 93, 095202 (2018)

51. Du, Z., Tian, B., Chai, H.P., Sun, Y., Zhao, X.H.: Rogue waves for the coupled variable-coefficient fourth-order nonlinear Schrödinger equations in an inhomogeneous optical fiber. Chaos Solitons Fractals 109, 90-98 (2018)

52. Yuan, Y.Q., Tian, B., Liu, L., Wu, X.Y., Sun, Y.: Solitons for the $(2+1)$-dimensional Konopelchenko-Dubrovsky equations. J. Math. Anal. Appl. 460, 476-486 (2018) 
53. Yuan, Y.Q., Tian, B., Sun, Y., Yin, H.M., Zhang, Z.: Mixed lump-stripe, bright rogue wave-stripe, dark rogue wave-stripe and dark rogue wave solutions of a generalized Kadomtsev-Petviashvili equation in fluid mechanics. Chin. J. Phys. 60 440-449 (2019)

54. Zhao, X.H., Tian, B., Xie, X.Y., Wu, X.Y., Sun, Y., Guo, Y.J.: Solitons, Bäcklund transformation and Lax pair for a $(2+1)$-dimensional Davey-Stewartson system on surface waves of finite depth. Waves Random Complex Media 28 , $356(2018)$

55. Gao, X.Y:: Mathematical view with observational/experimental consideration on certain $(2+1)$-dimensional waves in the cosmic/laboratory dusty plasmas. Appl. Math. Lett. 91, 165-172 (2019)

56. Wu, G.Z., Yu, L.J., Wang, Y.Y.: Fractional optical solitons of the space-time fractional nonlinear Schrödinger equation. Optik 207, $164405(2020)$

57. Yan, Y., Liu, W.: Stable transmission of solitons in the complex cubic-quintic Ginzburg-Landau equation with nonlinear gain and higher-order effects. Appl. Math. Lett. 98, 171-176 (2019)

58. Chen, S.J., Lin, J.N., Wang, Y.Y.: Soliton solutions and their stabilities of three $(2+1)$-dimensional $\mathfrak{P} \mathfrak{L}$-symmetric nonlinear Schrödinger equations with higher-order diffraction and nonlinearities. Optik 194, 162753 (2019)

59. Dai, C.Q., Wang, Y.Y., Fan, Y., Zhang, J.F.: Interactions between exotic multi-valued solitons of the $(2+1)$-dimensional Korteweg-de Vries equation describing shallow water wave. Appl. Math. Model. 80, 506-515 (2020)

60. Dai, C.Q., Fan, Y., Zhang, N.: Re-observation on localized waves constructed by variable separation solutions of $(1+1)$-dimensional coupled integrable dispersionless equations via the projective Riccati equation method. Appl. Math. Lett. 96, 20-26 (2019)

61. Liu, W., Zheng, X., Li, X.: Bright and dark soliton solutions to the partial reverse space-time nonlocal Mel'nikov equation. Nonlinear Dyn. (2018). https://doi.org/10.1007/s11071-018-4482-9

62. Fokou, M., Kofane, T.C., Mohamadou, A., Yomba, E.: Two-dimensional third- and fifth-order nonlinear evolution equations for shallow water waves with surface tension. Nonlinear Dyn. (2017). https://doi.org/10.1007/s11071-017-3938-7

\section{Submit your manuscript to a SpringerOpen ${ }^{\circ}$ journal and benefit from:}

- Convenient online submission

- Rigorous peer review

- Open access: articles freely available online

- High visibility within the field

- Retaining the copyright to your article

Submit your next manuscript at $\boldsymbol{\nabla}$ springeropen.com 